\title{
Multiscale computation of isotropic homogeneous turbulent flow
}

\author{
Tom Hou, Danping Yang, and Hongyu Ran
}

\begin{abstract}
In this article we perform a systematic multi-scale analysis and computation for incompressible Euler equations and Navier-Stokes Equations in both $2 \mathrm{D}$ and $3 \mathrm{D}$. The initial condition for velocity field has multiple length scales. By reparameterizing them in the Fourier space, we can formally organize the initial condition into two scales with the fast scale component being periodic. By making an appropriate multiscale expansion for the velocity field, we show that the two-scale structure is preserved dynamically. Moreover, we derive a well-posed homogenized equation for the incompressible Euler equations in the Eulerian formulations. Numerical experiments are presented to demonstrate that the homogenized equations indeed capture the correct averaged solution of the incompressible Euler and Navier Stokes equations. Moreover, our multiscale analysis reveals some interesting structure for the Reynolds stress terms, which provides a theoretical base for establishing an effective LES type of model for incompressible fluid flows.
\end{abstract}

\section{Introduction}

In this paper, we review some of our recent efforts in developing a systematic multiscale analysis for the incompressible Euler equations with highly oscillating initial velocity field. The understanding of scale interactions for incompressible flows at high Reynolds number has been a major challenge. For high Reynolds number flows, the degrees of freedom are so large that it is almost impossible to resolve all small scales by direct numerical simulations. From the view point of engineering applications, it is important to derive an effective equation that governs the large scale solution. The nonlinear and nonlocal nature of the incompressible Euler or Navier-Stokes equations makes it difficult to obtain a closure for a multiscale analysis.

1991 Mathematics Subject Classification. Primary 54C40, 14E20; Secondary 46E25, 20 C20. Key words and phrases. Multiscale analysis, Euler equations, turbulence.

The first and the third author was supported in part by an NSF grant DMS-0073916 and an NSF ITR grant ACI-0204932.

The second author was supported in part by an NSF grant DMS-0073916, the National Basic Research Program of P. R. China under the grant 2005CB321703, and Natural Science Foundation of China under the Grant 10441005, 10571108. 
One of the main challenges in performing multiscale analysis for incompressible flows is to understand how small scales propagate in time and whether the multiscale structure of the solution is preserved dynamically. The earliest effort in studying homogenization of the incompressible Euler equations was by McLaughlin, Papanicolaou, and Pironneau (MPP for short) in 1985 [6]. One of the key observations in MPP's work is that the oscillation is convected by the mean velocity field. By introducing a phase function $\boldsymbol{\theta}$, which is convected by the mean velocity field, they characterize the propagation of the small scale by the fast spatial variable, $\mathbf{y}=\boldsymbol{\theta} / \epsilon$. In addition, a fast time variable $\tau=t / \epsilon$ is introduced. However, the resulting cell problem with periodic boundary condition in both $\mathbf{y}$ and $\tau$ may not have a unique solution. Additional assumptions were imposed on the solution of the cell problem in order to derive a variant of the $k-\epsilon$ model.

In [10], we study the homogenization of the Euler equation using a full Lagrangian formulation. The multiscale structure of the solution becomes apparent in the Lagrangian formulation. Using the Lagrangian formulation, we can show that vorticity preserves naturally the multiscale structure of its initial data. Velocity can be constructed using the vorticity-stream function formulation. Thus, we turn a convection dominated transport problem into an elliptic problem for the stream function. Traditional multiscale analysis for elliptic problems $[\mathbf{1}]$ can be used to obtain a multiscale expansion for the stream function. At the end, we derive a well-posed multiscale system for the Lagrangian flow map and the stream function.

Based on our multiscale analysis in the Lagrangian formulation, we derive the corresponding homogenized equations for the Euler equations in the Eulerian formulation for velocity and pressure. The homogenized equation for the large scale velocity field has a similar structure as the Large Eddy Simulation model with a Reynolds stress term [7]. The small scale solution is governed by a cell problem using the stream function-vorticity formulation. By exploring the solution structure of the cell problem, we reveal some intrinsic structure of the Reynolds stress term. Our study also shows that the effect of the Reynolds stress term in the 2-D Euler equations is very different from the Reynolds stress for the 3-D Euler equations. We also explore the structure of the Reynolds stress term in developing a systematic Large Eddy Simulation model for incompressible flows.

We have performed a careful numerical study to confirm the convergence of our multiscale analysis in both two and three space dimensions. We choose an initial condition which is random and contains many scales. We compare the numerical simulation obtained by solving the homogenized equations with the well-resolved numerical simulation. We compute the solution for a relatively long time until the flow is sufficiently mixed and some large coherent structure emerges. Our numerical study demonstrates that the numerical simulation obtained by solving the homogenized equations captures very well the large scale feature as well as the small scale details of the well-resolved solution. Moreover, we obtain very good agreements with the well-resolved solution in terms of the Fourier spectra, the mean kinetic energy, and the enstrophy. The organization of the rest of the paper is as follows. In Section 2, we will present the formulation of the incompressible Euler equations with rapidly oscillating initial data and motivate our multiscale analysis. Section 3 is devoted to developing the two-scale analysis of the 3-D Euler equations in both Lagrangian and Eulerian formulations. In section 4, we study some intrinsic 
structure of the Reynolds stress term for the 3-D Euler equations. In Section 5, we will perform some numerical experiments to confirm our analytical results.

\section{Formulation}

We consider incompressible d-dimensional Euler equations with highly oscillating initial velocity field:

(a) $\mathbf{u}_{t}^{\epsilon}+\left(\mathbf{u}^{\epsilon} \cdot \nabla\right) \mathbf{u}^{\epsilon}+\nabla p^{\epsilon}=\mathbf{0}, \quad \forall t>0, \quad \mathbf{x} \in R^{d}$;

(b) $\nabla \cdot \mathbf{u}^{\epsilon}=0, \quad \forall t>0, \quad \mathbf{x} \in R^{d}$;

(c) $\mathbf{u}^{\epsilon}=\mathbf{U}(\mathbf{x})+\mathbf{W}\left(\mathbf{x}, \frac{\mathbf{x}}{\epsilon}\right), \quad t=0, \quad \mathbf{x} \in R^{d}$;

where $\mathrm{d}=2$ or $3, \mathbf{u}^{\epsilon}(t, \mathbf{x})$ and $p^{\epsilon}(t, \mathbf{x})$ are the velocity and the pressure fields respectively. We assume that $\mathbf{U}$ is a given smooth mean velocity field in $R^{d}$ and $\mathbf{W}(\mathbf{x}, \mathbf{y})$ is the oscillatory component of the velocity field which is a smooth function of $\mathbf{x}$ and $\mathbf{y}$, and is periodic in $\mathbf{y}$ with period 1 in each dimension. Moreover, we assume that $\mathbf{W}$ has zero mean

$$
\langle\mathbf{W}\rangle \equiv \int_{[0,1]^{d}} \mathbf{W}(\mathbf{x}, \mathbf{y}) d \mathbf{y}=\mathbf{0} .
$$

We do not consider the boundary effect in our study. The velocity field is assumed to be at rest at infinity. The question of interest is how to derive a homogenized equation for the averaged velocity field.

The homogenization theory of the Euler equations with oscillating data was first studied by McLaughlin-Papanicolaou-Pironneau (MPP for short) in 1985 [6]. To construct a multiscale expansion for the Euler equations, they made an important assumption that the oscillation is convected by the mean flow:

$$
\begin{aligned}
& \mathbf{u}^{\epsilon}(t, \mathbf{x})=\mathbf{u}(t, \mathbf{x})+\mathbf{w}\left(t, \mathbf{x}, \frac{t}{\epsilon}, \frac{\boldsymbol{\theta}(t, \mathbf{x})}{\epsilon}\right)+\epsilon \mathbf{u}_{1}\left(t, \mathbf{x}, \frac{t}{\epsilon}, \frac{\boldsymbol{\theta}(t, \mathbf{x})}{\epsilon}\right)+\cdots \cdots \\
& p^{\epsilon}(\mathbf{x}, t)=p(t, \mathbf{x})+q\left(t, \mathbf{x}, \frac{t}{\epsilon}, \frac{\boldsymbol{\theta}(t, \mathbf{x})}{\epsilon}\right)+\epsilon p_{1}\left(t, \mathbf{x}, \frac{t}{\epsilon}, \frac{\boldsymbol{\theta}(t, \mathbf{x})}{\epsilon}\right)+\cdots \cdots
\end{aligned}
$$

where $\mathbf{w}(t, \mathbf{x}, \tau, \mathbf{y}), \mathbf{u}_{1}(t, \mathbf{x}, \tau, \mathbf{y}), q(t, \mathbf{x}, \tau, \mathbf{y})$ and $p_{1}(t, \mathbf{x}, \tau, \mathbf{y})$ are assumed to be periodic in $\mathbf{y}$ and $\tau$ with zero mean, and the phase $\boldsymbol{\theta}$ is convected by the mean velocity field $\mathbf{u}$ :

$$
\frac{\partial \boldsymbol{\theta}}{\partial t}+\mathbf{u} \cdot \nabla \boldsymbol{\theta}=\mathbf{0}, \quad \boldsymbol{\theta}(0, \mathbf{x})=\mathbf{x} .
$$

Using multiscale expansion techniques, MPP obtained a periodic cell problem for $\mathbf{w}, \mathbf{u}_{1}, q$ and $p_{1}$. However, it is not clear whether the resulting cell problem for $\mathbf{w}(t, \mathbf{x}, \tau, \mathbf{y})$, etc has solution that is periodic in both $\mathbf{y}$ and $\tau$. Or if such solution exists, whether or not the solution is unique. Additional assumptions were imposed on the solution of the cell problem in order to derive a variant of the $k-\epsilon$ model.

Our study shows that the small scale oscillations are actually convected by the average of the full oscillatory velocity field, which, generally saying, is not solution of the equations (2.3). The oscillation in the flow map could have an order one contribution to the mean velocity field. More specifically, we define the inverse of 
the flow map, $\theta^{\epsilon}$, as follows:

$$
\begin{aligned}
& \text { (a) } \frac{\partial \boldsymbol{\theta}^{\epsilon}}{\partial t}+\left(\mathbf{u}^{\epsilon} \cdot \nabla\right) \boldsymbol{\theta}^{\epsilon}=\mathbf{0}, \quad \forall t>0, \quad \mathbf{x} \in R^{d} \\
& \text { (b) } \boldsymbol{\theta}^{\epsilon}=\mathbf{x}, \quad t=0, \quad \mathbf{x} \in R^{d} .
\end{aligned}
$$

Using the inverse of the flow map, we can characterize the evolution of the small scale vorticity field. Recall that the vorticity is defined as the curl of the velocity.

In $2-\mathrm{D}$, the vorticity becomes a scalar, $\omega^{\epsilon}=\partial_{x_{1}} u_{2}^{\epsilon}-\partial_{x_{2}} u_{1}^{\epsilon}$. For $2-\mathrm{D}$ incompressible inviscid flows, vorticity is conserved in time,

$$
\begin{aligned}
& \text { (a) } \omega_{t}^{\epsilon}+\mathbf{u}^{\epsilon} \cdot \nabla \omega^{\epsilon}=0, \\
& \text { (b) }\left.\omega\right|_{t=0}=\omega_{0},
\end{aligned}
$$

where $\omega_{0}(\mathbf{x}, \mathbf{x} / \epsilon)$ is the initial vorticity. Thus we have

$$
\omega^{\epsilon}(t, \mathbf{x})=\omega_{0}\left(\boldsymbol{\theta}^{\epsilon}(t, \mathbf{x}), \frac{\boldsymbol{\theta}^{\epsilon}(t, \mathbf{x})}{\epsilon}\right) .
$$

In 3-D Euler equations, the vorticity, defined as $\boldsymbol{\omega}^{\epsilon}=\nabla \times \mathbf{u}^{\epsilon}$, is no longer conserved in time due to vortex stretching. The vorticity equation now becomes

$$
\begin{aligned}
& \text { (a) } \boldsymbol{\omega}_{t}^{\epsilon}+\mathbf{u}^{\epsilon} \cdot \nabla \boldsymbol{\omega}^{\epsilon}=\boldsymbol{\omega}^{\epsilon} \cdot \nabla \mathbf{u}^{\epsilon}, \\
& \text { (b) }\left.\boldsymbol{\omega}\right|_{t=0}=\omega_{0},
\end{aligned}
$$

where $\omega_{0}(\mathbf{x}, \mathbf{x} / \epsilon)$ is the initial vorticity. In this case, we still have a relatively simple expression of vorticity in terms of the $\boldsymbol{\theta}^{\epsilon}$ variable [2]:

$$
\boldsymbol{\omega}^{\epsilon}(t, \mathbf{x})=\left(D \boldsymbol{\theta}^{\epsilon}\right)^{-1} \boldsymbol{\omega}_{0}\left(\boldsymbol{\theta}^{\epsilon}(t, \mathbf{x}), \frac{\boldsymbol{\theta}^{\epsilon}(t, \mathbf{x})}{\epsilon}\right),
$$

where $D \boldsymbol{\theta}^{\epsilon}$ is the Jacobian matrix of $\boldsymbol{\theta}^{\epsilon}$. It is clear from the vorticity formulation that the small scale information is propagated along the characteristic variable, $\boldsymbol{\theta}^{\epsilon}$. It is not an easy task to characterize the multiscale structure of $\boldsymbol{\theta}^{\epsilon}(t, \mathbf{x})$ since its multiscale structure is coupled to that of $\mathbf{u}^{\epsilon}$. However, it is clear from (2.6) and (2.8) that the small scale structure in $\boldsymbol{\theta}^{\epsilon}$ could have $O(1)$ contribution to the mean velocity field $\mathbf{u}$.

\section{Multiscale Analysis for 3D Euler Equations}

From the discussion in Section 2, we know that the small scale component of the vorticity field is propagated along the characteristic variable, $\boldsymbol{\theta}^{\epsilon}$. Thus it is natural to use the Lagrangian variable and the vorticity stream function formulation to perform the multiscale analysis for the 3-D incompressible Euler equations.

3.1. Vorticity-Stream Function Formulation. With the vorticity, the stream function, denoted by $\psi^{\epsilon}$, satisfies the following elliptic equation:

$$
-\Delta \psi^{\epsilon}=\omega^{\epsilon} .
$$

The velocity field can be constructed from the stream function:

$$
\mathbf{u}^{\epsilon}=\nabla \times \psi^{\epsilon}
$$

It follows from (2.8) that

$$
-\Delta \boldsymbol{\psi}^{\epsilon}=\left(D \boldsymbol{\theta}^{\epsilon}\right)^{-1} \boldsymbol{\omega}_{0}\left(\boldsymbol{\theta}^{\epsilon}, \frac{\boldsymbol{\theta}^{\epsilon}}{\epsilon}\right)
$$


One of the important observations we made in section 2 is that small scale information is propagated along the inverse of the flow map. Thus we look for the velocity field and the pressure of the following form:

$$
\mathbf{u}^{\epsilon}(t, \mathbf{x})=\mathbf{u}^{\epsilon}\left(t, \boldsymbol{\theta}^{\epsilon}, \frac{t}{\epsilon}, \frac{\boldsymbol{\theta}^{\epsilon}}{\epsilon}\right), \quad p^{\epsilon}(t, \mathbf{x})=p^{\epsilon}\left(t, \boldsymbol{\theta}^{\epsilon}, \frac{t}{\epsilon}, \frac{\boldsymbol{\theta}^{\epsilon}}{\epsilon}\right) .
$$

Here, the multiscale structure of the velocity field $\mathbf{u}^{\epsilon}$ is now coupled to the small scale structure of $\boldsymbol{\theta}^{\epsilon}(t, \mathbf{x})$. As a first step, we need to understand how small scales of $\boldsymbol{\theta}^{\epsilon}$ propagate in time. Naively, one may be tempted to assume that $\boldsymbol{\theta}^{\epsilon}$ has the following multiscale scale expansion:

$$
\boldsymbol{\theta}^{\epsilon}(t, \mathbf{x})=\boldsymbol{\theta}(t, \mathbf{x})+\epsilon \boldsymbol{\theta}^{(1)}\left(t, \mathbf{x}, \frac{t}{\epsilon}, \frac{\mathbf{x}}{\epsilon}\right)+\mathbf{O}\left(\epsilon^{2}\right)
$$

This is not correct. Under this assumption, $\boldsymbol{\theta}^{\epsilon}$ could develop infinitely many scales in powers of $\epsilon$ for $t>0$. By working on the Lagrangian flow map, we can show that the $\boldsymbol{\theta}^{\epsilon}$ is defined implicitly as follows [10]

$$
\boldsymbol{\theta}^{\epsilon}=\boldsymbol{\theta}(t, \mathbf{x})+\epsilon\left[\overline{\boldsymbol{\theta}}^{(1)}\left(t, \boldsymbol{\theta}, \frac{t}{\epsilon}\right)+\boldsymbol{\theta}^{(1)}\left(t, \boldsymbol{\theta}, \frac{t}{\epsilon}, \frac{\boldsymbol{\theta}^{\epsilon}}{\epsilon}\right)\right]+\cdots \cdots
$$

By combining all the fluctuation terms into two terms, we obtain the multi-scale expansion for $\boldsymbol{\theta}^{\epsilon}$ in the the form:

$$
\boldsymbol{\theta}^{\epsilon}=\boldsymbol{\theta}(t, \mathbf{x}, \tau)+\epsilon \boldsymbol{\Theta}(t, \boldsymbol{\theta}, \tau, \mathbf{y}),
$$

where $\tau=t / \epsilon$ and $\mathbf{y}=\boldsymbol{\theta}^{\epsilon} / \epsilon, \boldsymbol{\theta}$ is the average of $\boldsymbol{\theta}^{\epsilon}$, and $\boldsymbol{\Theta}$ is periodic in $\mathbf{y}$ with zero mean.

With this expansion, we can derive the homogenized equations in the vorticitystream function formulation: However, to get the first order correction to the stream function from the cell equations, we must solve an elliptic differential equation with varying coefficient. To simplify the computation, we introduce a change of variables from $\mathbf{y}$ to $\mathbf{z}$ as follows:

$$
\mathbf{z}=\mathbf{y}-\boldsymbol{\Theta}(t, \boldsymbol{\theta}, \tau, \mathbf{y}) \equiv G(\mathbf{y}), \quad \mathbf{y}=G^{-1}(\mathbf{z}),
$$

where $t, \boldsymbol{\theta}$, and $\tau$ are considered as parameters. Let $\widehat{g}=g(\mathbf{y})=g \circ G^{-1}(\mathbf{z})$. It is easy to show that $\widehat{g}$ is also a 1-periodic function in $\mathbf{z}$. Let us consider the geometric meaning of the transformation of (3.6). From the expansion (3.5), it is clear that $\mathbf{z}=\boldsymbol{\theta} / \epsilon$, since $\mathbf{y}=\boldsymbol{\theta}^{\epsilon} / \epsilon$. This implies that

$$
\widehat{\boldsymbol{\Theta}}=\widehat{\Theta}\left(t, \boldsymbol{\theta}, \tau, \frac{\boldsymbol{\theta}}{\epsilon}\right)
$$

where $\widehat{\boldsymbol{\Theta}}=\boldsymbol{\Theta}(\mathbf{y})=\mathbf{\Theta} \circ G^{-1}(\mathbf{z})$ by definition.

Based upon this observation, we look for multiscale expansion for $\boldsymbol{\theta}^{\epsilon}$ and $\psi^{\epsilon}$ of the form:

$$
\text { (a) } \boldsymbol{\theta}^{\epsilon}=\boldsymbol{\theta}(t, \mathbf{x}, \tau)+\epsilon \boldsymbol{\Theta}(t, \boldsymbol{\theta}, \tau, \mathbf{z})
$$$$
\text { (b) } \boldsymbol{\psi}^{\epsilon}=\boldsymbol{\psi}(t, \mathbf{x}, \tau)+\epsilon \boldsymbol{\Psi}(t, \boldsymbol{\theta}, \tau, \mathbf{z})
$$

where the fast variable $\mathbf{z}$ is now given by

$$
\mathbf{z}=\frac{\boldsymbol{\theta}}{\epsilon}
$$

and $\boldsymbol{\theta}$ and $\psi$ are averages of $\boldsymbol{\theta}^{\epsilon}$ and $\psi^{\epsilon}$ with respect to $\mathbf{z}$ over one period, and $\boldsymbol{\Theta}$ and $\boldsymbol{\Psi}$ are 1-periodic functions with zero mean in $\mathbf{z}$. 
Let $\nabla_{x} \times$ and $\nabla_{z} \times$ denote the curl operators with respect to variables $\mathbf{x}$ and $\mathbf{z}$, respectively. By performing a careful multiscale analysis, we can obtain the homogenized equations for both two and three dimensional Euler equations. Detailed multiscale analysis for the 3-D Euler equations can be found in [9]. We will summarize the main result in the next subsection.

3.2. Homogenized Equations and the Cell Problem. By using streamline function $\psi^{\epsilon}$ and Lagrangian map $\boldsymbol{\theta}^{\epsilon}$ given by (3.8), we have

$$
\mathbf{u}^{\epsilon}=\nabla_{x} \times \boldsymbol{\psi}+\left(D_{x} \boldsymbol{\theta}^{\top} \nabla_{z}\right) \times \boldsymbol{\Psi}+\epsilon \nabla_{x} \times \boldsymbol{\Psi} .
$$

Based on the expansions in (3.8) and (3.10), we look for expansion of $\left(\mathbf{u}^{\epsilon}, p^{\epsilon}\right)$ in the form:

$$
\begin{aligned}
& \text { (a) } \mathbf{u}^{\epsilon}=\mathbf{u}(t, \mathbf{x}, \tau)+\mathbf{w}(t, \boldsymbol{\theta}, \tau, \mathbf{z}) \\
& \text { (b) } p^{\epsilon}=p(t, \mathbf{x}, \tau)+q(t, \boldsymbol{\theta}, \tau, \mathbf{z})
\end{aligned}
$$

where $(\mathbf{w}, q)$ are the total fluctuating components including higher-order terms and are periodic functions in $\mathbf{z}$ with zero means. Similarly, $(\mathbf{u}, p)$ are the total mean components including higher-order terms.

Assume that the solutions of the Euler equations (2.1) and the transport equation (2.4) governing $\boldsymbol{\theta}^{\epsilon}$ have a multiscale expansion of the forms (3.11) and (3.8). Let $\bar{\partial}_{t}=\partial_{t}+\epsilon^{-1} \partial_{\tau}$ be the total derivative in time. We have the following results:

Averaged equations for the velocity:

$$
\begin{aligned}
& \text { (a) } \bar{\partial}_{t} \mathbf{u}+\left(\mathbf{u} \cdot \nabla_{x}\right) \mathbf{u}+\nabla_{x} p+\nabla_{x} \cdot\langle\mathbf{w} \otimes \mathbf{w}\rangle=\mathbf{0} \\
& \text { (b) } \nabla_{x} \cdot \mathbf{u}=0 \\
& \text { (c) }\left.\mathbf{u}\right|_{t=0}=\mathbf{U}(\mathbf{x})
\end{aligned}
$$

where $\mathbf{w} \otimes \mathbf{w}=\mathbf{w w}^{\top}$.

Averaged equations for the inverse of the Lagrangian map:

$$
\begin{aligned}
& \text { (a) } \bar{\partial}_{t} \boldsymbol{\theta}+\left(\mathbf{u} \cdot \nabla_{x}\right) \boldsymbol{\theta}+\epsilon \nabla_{x} \cdot\langle\boldsymbol{\Theta} \otimes \mathbf{w}\rangle=\mathbf{0}, \\
& \text { (b) }\left.\boldsymbol{\theta}\right|_{t=0}=\mathbf{x} .
\end{aligned}
$$

Furthermore, assume that $(\mathbf{w}, q)$ and $\Theta$ are of form

(a) $\mathbf{w}=\mathbf{w}^{(0)}(t, \boldsymbol{\theta}, \tau, \mathbf{z})+\epsilon \mathbf{w}^{(1)}(t, \boldsymbol{\theta}, \tau, \mathbf{z})+\cdots \cdots$

(b) $q=q^{(0)}(t, \boldsymbol{\theta}, \tau, \mathbf{z})+\epsilon q^{(1)}(t, \boldsymbol{\theta}, \tau, \mathbf{z})+\cdots \cdots$,

(c) $\boldsymbol{\Theta}=\boldsymbol{\Theta}^{(0)}(t, \boldsymbol{\theta}, \tau, \mathbf{z})+\epsilon \boldsymbol{\Theta}^{(1)}(t, \boldsymbol{\theta}, \tau, \mathbf{z})+\cdots \cdots$.

We have the following cell problem for the first two terms.

Leading order equations for the fluctuation of the velocity:

(a) $\partial_{\tau} \mathbf{w}^{(0)}+D_{z} \mathbf{w}^{(0)} D_{x} \boldsymbol{\theta} \mathbf{w}^{(0)}+D_{x} \boldsymbol{\theta}^{\top} \nabla_{z} q^{(0)}=\mathbf{0}$

(b) $\left(D_{x} \boldsymbol{\theta}^{\top} \nabla_{z}\right) \cdot \mathbf{w}^{(0)}=0$,

(c) $\left.\mathbf{w}^{(0)}\right|_{\tau=t=0}=\mathbf{W}$. 
First order correction equations for the fluctuation of the velocity:

$$
\begin{aligned}
& \text { (a) } \partial_{\tau} \mathbf{w}^{(1)}+D_{z} \mathbf{w}^{(1)} D_{x} \boldsymbol{\theta} \mathbf{w}^{(0)}+D_{z} \mathbf{w}^{(0)} D_{x} \boldsymbol{\theta} \mathbf{w}^{(1)}+D_{x} \boldsymbol{\theta}^{\top} \nabla_{z} q^{(1)} \\
& =-\left[\partial_{t} \mathbf{w}^{(0)}+D_{x} \mathbf{w}^{(0)} \mathbf{w}^{(0)}+\nabla_{x} q^{(0)}+\left(\mathbf{w}^{(0)} \cdot \nabla_{x}\right) \mathbf{u}\right. \\
& \left.-\nabla_{\boldsymbol{x}} \cdot\left\langle\mathbf{w}^{(0)} \otimes \mathbf{w}^{(0)}\right\rangle-D_{z} \mathbf{w}^{(0)} \nabla_{x} \cdot\left\langle\Theta^{(1)} \otimes \mathbf{w}^{(0)}\right\rangle\right] \\
& \text { (b) }\left(D_{x} \boldsymbol{\theta}^{\top} \nabla_{z}\right) \cdot \mathbf{w}^{(1)}=-\nabla_{x} \cdot \mathbf{w}^{(0)} \text {, } \\
& \text { (c) }\left.\mathbf{w}^{(1)}\right|_{\tau=t=0}=\mathbf{0}
\end{aligned}
$$

First order correction equations for the fluctuation of the inverse of the Lagrangian map:

$$
\begin{aligned}
& \text { (a) } \partial_{\tau} \Theta^{(1)}+\left(I+D_{z} \Theta^{(1)}\right) D_{x} \boldsymbol{\theta} \mathbf{w}^{(0)}=\mathbf{0} \\
& \text { (b) }\left.\Theta^{(1)}\right|_{\tau=t=0}=\mathbf{0} .
\end{aligned}
$$

We remark that there are several differences between our homogenized equations (3.12)-(3.17) and thsoe obtained by MPP in [6]. The first one is that no periodic structure in $\tau$ is assumed. In general, there may not exist a periodic structure in the fast time variable $\tau$. The periodic assumption on $\tau$ leads to difficulties in both theoretical study and practical computations. The second one is that the periodic structure of the small scales is propagated along the mean of the Lagrangian map. Further discussions can be found from [10] where we derive the homogenization of the $2 \mathrm{D}$ Euler equations in great details.

The additional term $\langle\mathbf{w} \otimes \mathbf{w}\rangle$ in the averaged velocity equation is referred to as the Reynolds stress. How to model Reynolds stress has fundamental and practical importance in engineering applications. It is very important to understand the structure of Reynolds stress in order to establish a Large Eddy Simulation (LES) model with some accuracy. Practical simulations show that the effect of higher order correction terms could not be ignored. The first order correction terms in our cell problem provide insight to the higher order correction terms in the Reynolds stress.

3.3. Expansion in the Lagrangian Formulation. To understand the structure of the Reynolds stress term in detail, we consider expansion in the Lagrangian formulation. For this purpose, we need to expand the Jacobian matrix, $B=$ $\left(D_{x} \boldsymbol{\theta}^{\epsilon}\right)^{-1}$. Note that

$$
\left(D_{x} \boldsymbol{\theta}^{\epsilon}\right)^{-1}=\left(\nabla_{x} \times\left(\theta_{2}^{\epsilon} \nabla_{x} \theta_{3}^{\epsilon}\right), \nabla_{x} \times\left(\theta_{3}^{\epsilon} \nabla_{x} \theta_{1}^{\epsilon}\right), \nabla_{x} \times\left(\theta_{1}^{\epsilon} \nabla_{x} \theta_{2}^{\epsilon}\right)\right)
$$

Let $B=\left(D_{x} \boldsymbol{\theta}^{\epsilon}\right)^{-1}$ and $\widetilde{\nabla}_{z}=D \boldsymbol{\theta}^{\top} \nabla_{z}$. Then we have

$$
B=\bar{H}+\widetilde{H}+\epsilon H^{(1)}+\epsilon^{2} H^{(2)}
$$

where

$$
\bar{H}=\left(\nabla_{x} \times\left(\theta_{2} \nabla_{x} \theta_{3}\right), \nabla_{x} \times\left(\theta_{3} \nabla_{x} \theta_{1}\right), \nabla_{x} \times\left(\theta_{1} \nabla_{x} \theta_{2}\right)\right)=\left|D_{x} \boldsymbol{\theta}\right| D_{x} \boldsymbol{\theta}^{-1}
$$


and

$$
\begin{aligned}
\widetilde{H} & =\left(\nabla_{x} \times\left(\theta_{2} \widetilde{\nabla}_{z} \Theta_{3}\right), \nabla_{x} \times\left(\theta_{3} \widetilde{\nabla}_{z} \Theta_{1}\right), \nabla_{x} \times\left(\theta_{1} \widetilde{\nabla}_{z} \Theta_{2}\right)\right) \\
& +\left(\widetilde{\nabla}_{z} \times\left(\Theta_{2} \nabla_{x} \theta_{3}\right), \widetilde{\nabla}_{z} \times\left(\Theta_{3} \nabla_{x} \theta_{1}\right), \widetilde{\nabla}_{z} \times\left(\Theta_{1} \nabla_{x} \theta_{2}\right)\right) \\
& +\left(\widetilde{\nabla}_{z} \times\left(\theta_{2} \nabla_{x} \Theta_{3}\right), \widetilde{\nabla}_{z} \times\left(\theta_{3} \nabla_{x} \Theta_{1}\right), \widetilde{\nabla}_{z} \times\left(\theta_{1} \nabla_{x} \Theta_{2}\right)\right) \\
& +\left(\widetilde{\nabla}_{z} \times\left(\Theta_{2} \widetilde{\nabla}_{z} \Theta_{3}\right), \widetilde{\nabla}_{z} \times\left(\Theta_{3} \widetilde{\nabla}_{z} \Theta_{1}\right), \widetilde{\nabla}_{z} \times\left(\Theta_{1} \widetilde{\nabla}_{z} \Theta_{2}\right)\right)
\end{aligned}
$$

and

$$
\begin{aligned}
H^{(1)} & =\left(\nabla_{x} \times\left(\Theta_{2} \nabla_{x} \theta_{3}\right), \nabla_{x} \times\left(\Theta_{3} \nabla_{x} \theta_{1}\right), \nabla_{x} \times\left(\Theta_{1} \nabla_{x} \theta_{2}\right)\right) \\
& +\left(\nabla_{x} \times\left(\theta_{2} \nabla_{x} \Theta_{3}\right), \nabla_{x} \times\left(\theta_{3} \nabla_{x} \Theta_{2}\right), \nabla_{x} \times\left(\theta_{1} \nabla_{x} \Theta_{2}\right)\right) \\
& +\left(\nabla_{x} \times\left(\Theta_{2} \widetilde{\nabla}_{z} \Theta_{3}\right), \nabla_{x} \times\left(\Theta_{3} \widetilde{\nabla}_{z} \Theta_{1}\right), \nabla_{x} \times\left(\Theta_{1} \widetilde{\nabla}_{z} \Theta_{2}\right)\right) \\
& +\left(\widetilde{\nabla}_{z} \times\left(\Theta_{2} \nabla_{x} \Theta_{3}\right), \widetilde{\nabla}_{z} \times\left(\Theta_{3} \nabla_{x} \Theta_{1}\right), \widetilde{\nabla}_{z} \times\left(\Theta_{1} \nabla_{x} \Theta_{2}\right)\right)
\end{aligned}
$$

and

$$
H^{(2)}=\left(\nabla_{x} \times\left(\Theta_{2} \nabla_{x} \Theta_{3}\right), \nabla_{x} \times\left(\Theta_{3} \nabla_{x} \Theta_{1}\right), \nabla_{x} \times\left(\Theta_{1} \nabla_{x} \Theta_{2}\right)\right)
$$

From the elliptic equation (3.3) for the streamline function, we obtain

$$
\begin{aligned}
- & \frac{1}{\epsilon} \nabla_{z} \cdot\left(D_{x} \boldsymbol{\theta} D_{x} \boldsymbol{\theta}^{\top} \nabla_{z} \boldsymbol{\Psi}\right)-\nabla_{z} \cdot\left(D_{x} \boldsymbol{\theta} \nabla_{x} \boldsymbol{\Psi}\right) \\
& -\nabla_{x} \cdot\left(D_{x} \boldsymbol{\theta}^{\top} \nabla_{z} \boldsymbol{\Psi}\right)-\Delta_{x}(\boldsymbol{\psi}+\epsilon \boldsymbol{\Psi}) \\
& =B \boldsymbol{\omega}_{0}(\boldsymbol{\theta}+\epsilon \boldsymbol{\Theta}, \mathbf{z}+\boldsymbol{\Theta}) .
\end{aligned}
$$

By equating the equations of the same power, we obtain the following homogenization result for $\boldsymbol{\psi}$ and $\boldsymbol{\Psi}$.

The averaged equations for $\psi$ :

$$
-\Delta_{x} \boldsymbol{\psi}=\left\langle\left(B \boldsymbol{\omega}_{0}(\boldsymbol{\theta}+\epsilon \boldsymbol{\Theta}, \mathbf{z}+\boldsymbol{\Theta})\right\rangle\right.
$$

The cell problem for the fluctuation $\boldsymbol{\Psi}$ :

$$
\begin{aligned}
&- \nabla_{z} \cdot\left(D_{x} \boldsymbol{\theta} D_{x} \boldsymbol{\theta}^{\top} \nabla_{z} \boldsymbol{\Psi}\right)-\epsilon \nabla_{z} \cdot\left(D_{x} \boldsymbol{\theta} \nabla_{x} \boldsymbol{\Psi}\right)-\epsilon \nabla_{x} \cdot\left(D_{x} \boldsymbol{\theta}^{\top} \nabla_{z} \boldsymbol{\Psi}\right)-\epsilon^{2} \Delta_{x} \boldsymbol{\Psi} \\
&=\epsilon\left[\left(B \boldsymbol{\omega}_{0}(\boldsymbol{\theta}+\epsilon \boldsymbol{\Theta}, \mathbf{z}+\boldsymbol{\Theta})-\left\langle B \boldsymbol{\omega}_{0}(\boldsymbol{\theta}+\epsilon \boldsymbol{\Theta}, \mathbf{z}+\boldsymbol{\Theta})\right\rangle\right] .\right.
\end{aligned}
$$

Furthermore, let

$$
\begin{aligned}
& \text { (a) } \boldsymbol{\psi}=\boldsymbol{\psi}^{(0)}+\epsilon \boldsymbol{\psi}^{(1)}+\cdots \cdots \\
& \text { (b) } \boldsymbol{\Psi}=\boldsymbol{\Psi}^{(0)}+\epsilon \boldsymbol{\Psi}^{(1)}+\cdots \cdots
\end{aligned}
$$

Then $\psi^{(0)}$ and $\Psi^{(0)}$ satisfy the following equations.

The homogenized equation:

$$
-\Delta_{x} \psi^{(0)}=\left\langle(\bar{H}+\widetilde{H}) \boldsymbol{\omega}_{0}\left(\boldsymbol{\theta}, \mathbf{z}+\boldsymbol{\theta}^{(1)}\right)\right\rangle .
$$

The cell problem:

$$
-\nabla_{z} \cdot\left(D_{x} \boldsymbol{\theta} D_{x} \boldsymbol{\theta}^{\top} \nabla_{z} \boldsymbol{\Psi}^{(0)}\right)=\tilde{H} \boldsymbol{\sigma}_{0}\left(\boldsymbol{\theta}, \mathbf{z}+\boldsymbol{\theta}^{(1)}\right)
$$

where $\sigma_{0}(\mathbf{x}, \mathbf{z})=\nabla_{z} \times \mathbf{W}(\mathbf{x}, \mathbf{z})$. 
First order correction equations for the fluctuation of the Lagrangian map:

$$
\begin{aligned}
& \text { (a) } \partial_{\tau} \boldsymbol{\theta}^{(1)}+\left(I+D_{z} \boldsymbol{\theta}^{(1)}\right)\left(D_{x} \overline{\boldsymbol{\theta}}\right) \mathbf{w}=\mathbf{0}, \\
& \text { (b) }\left.\boldsymbol{\theta}^{(1)}\right|_{\tau=t=0}=\mathbf{0} .
\end{aligned}
$$

The leading order term, $\mathbf{u}^{(0)}$ and $\mathbf{w}^{(0)}$, of the velocity field is given by

$$
\mathbf{u}^{(0)}=\nabla_{x} \times \boldsymbol{\psi}^{(0)}, \quad \mathbf{w}^{(0)}=\left(D_{x} \boldsymbol{\theta}^{\top} \nabla_{z}\right) \times \boldsymbol{\Psi}^{(1)} .
$$

\section{The Structure of the Reynolds Stress}

The structure of the Reynolds stress term $\langle\mathbf{w} \otimes \mathbf{w}\rangle$ is most interesting in both engineering applications and theoretical study. Many LES models have been proposed to simulate turbulent flows. Our multiscale analysis reveals some interesting structures of the Reynolds stress term. Our hope is that by exploring these structures, one may establish a more systematic LES model to model the large scale solution of the incompressible flow.

From the multiscale analysis presented earlier, we see that the leading order term, $\left\langle\mathbf{w}^{(0)} \otimes \mathbf{w}^{(0)}\right\rangle$, of the Reynolds stress term is of the form

$$
\left\langle\mathbf{w}^{(0)} \otimes \mathbf{w}^{(0)}\right\rangle=\left\langle\left(D_{x} \boldsymbol{\theta}^{\top} \nabla_{z}\right) \times \boldsymbol{\Psi}^{(1)} \otimes\left(D_{x} \boldsymbol{\theta}^{\top} \nabla_{z}\right) \times \boldsymbol{\Psi}^{(1)}\right\rangle,
$$

where $\mathbf{w}^{(0)}$ depends mainly on the oscillating part $\mathbf{W}$ of the initial velocity. Our computational results show that, as time increases, the divergence of $\left\langle\mathbf{w}^{(0)} \otimes \mathbf{w}^{(0)}\right\rangle$, $\nabla_{x} \cdot\left\langle\mathbf{w}^{(0)} \otimes \mathbf{w}^{(0)}\right\rangle$, becomes smaller and smaller, although $\left\langle\mathbf{w}^{(0)} \otimes \mathbf{w}^{(0)}\right\rangle$ itself may not be very small. On the other hand, we observe that the contribution from the small scales that are generated dynamically from nonlinear interaction between large scales and small scales or among large scales themselves becomes larger and its effect plays a more important role. In fact, even in the case when the initial condition has no small scales, small scales can still be generated dynamically and become stronger and stranger. In this case, the effect of higher order correction terms can not be ignored. We remark that the parameter $\epsilon$ is not very small in practice and therefore we can not neglect the contributions from the first order correction terms.

Based on the above discussions, we should take into account the higher order correction terms in the Reynolds stress, especially those terms that account for the small scales that are generated from nonlinear interaction dynamically. Let

$$
\mathbf{g}=\frac{1}{\epsilon} \nabla_{z} \times \mathbf{W}(\mathbf{x}, \mathbf{z})+\nabla_{x} \times \mathbf{W}(\mathbf{x}, \mathbf{z}), \quad \mathbf{h}=\nabla_{x} \times \mathbf{U}(\mathbf{x}) .
$$

The initial vorticity reads

$$
\boldsymbol{\omega}_{0}=\mathbf{g}(\mathbf{x}, \mathbf{z})+\mathbf{h}(\mathbf{x})
$$

It is clear that $\mathbf{g}$ results from the initial fluctuation $\mathbf{W}$ and $\mathbf{h}$ from the initial mean velocity. We can write the vorticity as

$$
\nabla \times \mathbf{u}^{\epsilon}=\left(\bar{H}+\widetilde{H}+\epsilon H^{(1)}+\epsilon^{2} H^{(2)}\right)[\mathbf{g}(\boldsymbol{\theta}+\epsilon \boldsymbol{\Theta}, \mathbf{z}+\boldsymbol{\Theta})+\mathbf{h}(\boldsymbol{\theta}+\epsilon \boldsymbol{\Theta})] .
$$

Since

$$
\mathbf{h}(\boldsymbol{\theta}+\epsilon \boldsymbol{\Theta})=\mathbf{h}(\boldsymbol{\theta})+\epsilon D_{\boldsymbol{\theta}} \mathbf{h}(\boldsymbol{\theta}) \boldsymbol{\Theta}+O\left(\epsilon^{2}\right),
$$


we have

$$
\begin{aligned}
\nabla \times \overline{\mathbf{u}} & =\bar{H} \mathbf{h}(\boldsymbol{\theta})+\epsilon\left\langle\widetilde{H} D_{\boldsymbol{\theta}} \mathbf{h}(\boldsymbol{\theta}) \boldsymbol{\Theta}\right\rangle \\
& +\left\langle\left(\bar{H}+\widetilde{H}+\epsilon H^{(1)}\right) \mathbf{g}(\boldsymbol{\theta}+\epsilon \boldsymbol{\Theta}, \mathbf{z}+\boldsymbol{\Theta})\right\rangle+O\left(\epsilon^{2}\right) .
\end{aligned}
$$

Thus, we can decompose the vorticity into two terms as follows:

$$
\nabla \times \widetilde{\mathbf{u}}=\widetilde{\boldsymbol{\sigma}}+\widetilde{H} \bar{H}^{-1} \nabla \times \overline{\mathbf{u}}
$$

with

$$
\begin{aligned}
\widetilde{\boldsymbol{\sigma}}= & \left(H+\widetilde{H}+\epsilon H^{(1)}\right) \mathbf{g}(\boldsymbol{\theta}+\epsilon \boldsymbol{\Theta}, \mathbf{z}+\boldsymbol{\Theta}) \\
& -\left\langle\left(H+\widetilde{H}+\epsilon H^{(1)}\right) \mathbf{g}(\boldsymbol{\theta}+\epsilon \boldsymbol{\Theta}, \mathbf{z}+\boldsymbol{\Theta})\right\rangle \\
& -\widetilde{H} \bar{H}^{-1}\left\langle\left(\bar{H}+\widetilde{H}+\epsilon H^{(1)}\right) \mathbf{g}(\boldsymbol{\theta}+\epsilon \boldsymbol{\Theta}, \mathbf{z}+\boldsymbol{\Theta})\right\rangle+\mathbf{O}\left(\epsilon^{2}\right) .
\end{aligned}
$$

Next, we decompose $\boldsymbol{\Psi}$ into two parts:

$$
\boldsymbol{\Psi}=\boldsymbol{\Psi}_{1}+\boldsymbol{\Psi}_{2}
$$

where $\boldsymbol{\Psi}_{1}$ satisfies

$$
\begin{aligned}
& \left.-\frac{1}{\epsilon} \nabla_{z} \cdot\left(D_{x} \boldsymbol{\theta} D_{x} \boldsymbol{\theta}^{\top} \nabla_{z} \boldsymbol{\Psi}_{1}\right)-\nabla_{z} \cdot\left(D_{x} \boldsymbol{\theta} \nabla_{x} \boldsymbol{\Psi}_{1}\right)\right) \\
& \quad-\nabla_{\boldsymbol{x}} \cdot\left(D_{x} \boldsymbol{\theta}^{\top} \nabla_{z} \boldsymbol{\Psi}_{1}\right)-\Delta_{x}\left(\boldsymbol{\psi}+\epsilon \boldsymbol{\Psi}_{1}\right) \\
& =\widetilde{\boldsymbol{\sigma}}
\end{aligned}
$$

and $\Psi_{2}$ satisfies

$$
\begin{aligned}
& \left.-\frac{1}{\epsilon} \nabla_{z} \cdot\left(D_{x} \boldsymbol{\theta} D_{x} \boldsymbol{\theta}^{\top} \nabla_{z} \boldsymbol{\Psi}_{2}\right)-\nabla_{z} \cdot\left(D_{x} \boldsymbol{\theta} \nabla_{x} \boldsymbol{\Psi}_{2}\right)\right) \\
& \quad-\nabla_{x} \cdot\left(D_{x} \boldsymbol{\theta}^{\top} \nabla_{z} \boldsymbol{\Psi}_{2}\right)-\Delta_{x}\left(\boldsymbol{\psi}+\epsilon \boldsymbol{\Psi}_{2}\right) \\
& \quad=\widetilde{H} \bar{H}^{-1} \nabla \times \overline{\mathbf{u}}
\end{aligned}
$$

Obviously, $\boldsymbol{\Psi}_{1}$ is dependent upon the initial fluctuation $\mathbf{W}$ and $\boldsymbol{\Psi}_{2}$ is driven by the averaging velocity field $\overline{\mathbf{u}}$.

Similarly, we decompose $\widetilde{\mathbf{u}}$ into two parts

$$
\mathbf{w}=\widetilde{\mathbf{w}}+\widetilde{\mathbf{v}}
$$

where

$$
\begin{aligned}
& \text { (a) } \tilde{\mathbf{w}}=\left(D_{x} \boldsymbol{\theta}^{\top} \nabla_{z}\right) \times \boldsymbol{\Psi}_{1}+\epsilon \nabla_{x} \times \boldsymbol{\Psi}_{1}, \\
& \text { (b) } \tilde{\mathbf{v}}=\left(D_{x} \boldsymbol{\theta}^{\top} \nabla_{z}\right) \times \boldsymbol{\Psi}_{2}+\epsilon \nabla_{x} \times \boldsymbol{\Psi}_{2} .
\end{aligned}
$$

By exploring the solution structure of $\boldsymbol{\Psi}_{2}$, we can further express $\widetilde{\mathbf{v}}$ as

$$
\left.\widetilde{\mathbf{v}}=\epsilon\left(D_{x} \boldsymbol{\theta}^{\top} \nabla_{z}\right) \times K_{3}(\widetilde{H}) H^{-1} \nabla_{x} \times \overline{\mathbf{u}}\right]+\mathbf{O}\left(\epsilon^{2}\right) .
$$

where $K_{3}$, as a solution operator $v=K_{3}(f)$, is defined by solving the following boundary value problem:

$$
-\nabla_{z} \cdot\left(D_{x} \boldsymbol{\theta} D_{x} \boldsymbol{\theta}^{\top} \nabla_{z} v\right)=f
$$

We obtain the following results. 
The Reynolds stress term satisfies the following approximation equation:

$$
\begin{aligned}
\nabla_{x} \cdot & \langle\mathbf{w} \otimes \mathbf{w}\rangle=\nabla_{x} \cdot\langle\widetilde{\mathbf{w}} \otimes \widetilde{\mathbf{w}}\rangle \\
& +\epsilon\left[\nabla_{x} \cdot\left\langle\left[\left(D_{x} \boldsymbol{\theta}^{\top} \nabla_{z}\right) \times\left(K_{3}(\widetilde{H}) \bar{H}^{-1} \nabla_{x} \times \overline{\mathbf{u}}\right)\right] \otimes \widetilde{\mathbf{w}}\right\rangle\right. \\
& \left.+\nabla_{x} \cdot\left\langle\widetilde{\mathbf{w}} \otimes\left[\left(D_{x} \boldsymbol{\theta}^{\top} \nabla_{z}\right) \times\left[K_{3}(\widetilde{H}) \bar{H}^{-1} \nabla_{x} \times \overline{\mathbf{u}}\right)\right]\right\rangle\right] \\
& +\epsilon^{2} \nabla_{x} \cdot\left\langle\left(D_{x} \boldsymbol{\theta}^{\top} \nabla_{z}\right) \times\left[K_{3}(\widetilde{H}) \bar{H}^{-1} \nabla_{x} \times \overline{\mathbf{u}}\right]\right. \\
& \left.\otimes\left(D_{x} \boldsymbol{\theta}^{\top} \nabla_{z}\right) \times\left[K_{3}(\widetilde{H}) \bar{H}^{-1} \nabla_{x} \times \overline{\mathbf{u}}\right]\right\rangle,
\end{aligned}
$$

where the truncation error is $\mathbf{O}\left(\epsilon^{3}\right)$, and $\widetilde{\mathbf{w}}$ is given by

$$
\tilde{\mathbf{w}}=\mathbf{w}^{(0)}+\epsilon \mathbf{w}^{(1)}+\cdots \cdots
$$

where $\mathbf{w}^{(0)}$ and $\mathbf{w}^{(1)}$ are defined by (3.15) and (3.16). $\bar{H}$ and $\widetilde{H}$ are defined by (3.20) and (3.21).

We remark that numerical experiments show that the terms in (4.12) have the same order in fully developed turbulent flows. For sufficiently mixed homogeneous fluid flows, some of the quantities in the Reynolds stress may become universal or slowly varying. If we can identify these quantities, we may need to solve one or several cell problems to get a good approximation of the Reynolds stress. This would provide a systematic way to derive a LES model.

4.1. Structure of the Reynolds Stress Term in the 2D Case. Before we end this section, we discuss briefly the structure of the Reynolds stress term for the 2D Euler equations. Let $\boldsymbol{\xi}=-D_{x} \boldsymbol{\theta}^{-1} \boldsymbol{\Theta}$ and $K_{2}$ be the solution operator $v=K_{2}(f)$ defined as the solution of the following boundary value problem:

$$
-\nabla_{z}^{\perp} \cdot\left(B^{\top} B \nabla_{z}^{\perp} v\right)=f
$$

We have

$$
\widetilde{\mathbf{w}}=\epsilon^{2} B \nabla_{z}^{\perp}\left[K_{2}(\boldsymbol{\xi})^{\top} \nabla_{x} \bar{\omega}\right]+\mathbf{O}\left(\epsilon^{3}\right)=\epsilon^{2} B A \nabla_{x} \bar{\omega}+\mathbf{O}\left(\epsilon^{3}\right)
$$

where

$$
A=\left(\nabla_{z}^{\perp}\left(B K\left(\xi_{1}\right)\right), \nabla_{z}^{\perp}\left(B K\left(\xi_{2}\right)\right)\right) .
$$

With the above information for the oscillatory component of the velocity field, we can now characterize the Reynolds stress term as follows:

$$
\begin{aligned}
\nabla_{x} \cdot & \langle\mathbf{w} \otimes \mathbf{w}\rangle=\nabla_{x} \cdot(\widetilde{\mathbf{w}} \otimes \widetilde{\mathbf{w}}) \\
& -\epsilon^{2}\left[\nabla_{x} \cdot\left\langle\widetilde{\mathbf{w}} \otimes\left(B A \nabla_{x} \bar{\omega}\right)\right\rangle+\nabla_{x} \cdot\left\langle\left(B A \nabla_{x} \bar{\omega}\right) \otimes \widetilde{\mathbf{w}}\right\rangle\right]+\mathbf{O}\left(\epsilon^{3}\right)
\end{aligned}
$$

where $\widetilde{\mathbf{w}}$ is given by

$$
\widetilde{\mathbf{w}}=\mathbf{w}^{(0)}+\epsilon \mathbf{w}^{(1)}+\cdots \cdots
$$

with $\mathbf{w}^{(0)}$ and $\mathbf{w}^{(1)}$ defined by (3.15) and (3.16).

For detailed derivation of the Reynolds stress, $\overline{\mathbf{u}}$ and $\widetilde{\mathbf{u}}^{(1)}$, we refer to [9]. 


\section{Numerical Experiments}

In previous sections we perform multiscale analysis on flows that the initial velocity field has two scales. However, practical fluid flows have infinitely nonseparable scales. We have developed a scheme to transform a general initial condition with many non-separable scales into the form of a two-scale function so that we can use the two-scale analysis developed earlier to study more general multiscale problems [10]. In this section, we will perform detailed numerical experiments to check the accuracy of the multiscale analysis in both two and three space dimensions.

5.1. Numerical Experiments for the 2D Navier-Stokes Equations. We test the multiscale model for 2-D incompressible flow in a doubly periodic box of size $2 \pi \times 2 \pi$. For the homogenized problem, we solve the homogenized Euler equations given in Section 4.1 of [10],

$$
\begin{aligned}
& \text { (a) } \bar{\partial}_{t} \overline{\mathbf{u}}+\left(\overline{\mathbf{u}} \cdot \nabla_{x}\right) \overline{\mathbf{u}}+\nabla_{x} \bar{p}+\nabla_{x} \cdot\langle\mathbf{w} \otimes \mathbf{w}\rangle=\nu \Delta \mathbf{u} \\
& \text { (b) } \nabla_{x} \cdot \overline{\mathbf{u}}=0 \\
& \text { (c) }\left.\overline{\mathbf{u}}\right|_{t=0}=\mathbf{U}
\end{aligned}
$$

where $\nu$ is viscosity. Since $\nu$ is very small, a minor modification to the multiscale analysis can be made to accommodate the small viscous effect in the homogenized equations. We choose $\nu=10^{-4}$ in our computations. Note that the viscous term only affects the mean velocity equation, and the cell problem is still inviscid.

For the cell problem, we solve the simplified equations,

$$
\begin{aligned}
& \text { (a) }-\nabla_{z}^{\perp} \cdot\left(D_{x} \theta^{-\top} D_{x} \theta^{-1} \nabla_{z}^{\perp} \psi^{(1)}\right)=\sigma_{0}\left(\theta, \mathbf{z}+\theta^{(1)}\right), \\
& \text { (b) } \sigma_{0}(\mathbf{x}, \mathbf{z})=\nabla_{z}^{\perp} \cdot \mathbf{W}(\mathbf{x}, \mathbf{z}) .
\end{aligned}
$$

where $\mathrm{z}$ is given by equation (3.6)

(a) $\partial_{\tau} \theta^{(1)}-\left(I+D_{z} \theta^{(1)}\right) \nabla_{z}^{\perp} \psi^{(1)}=\mathbf{0}$

(b) $\left.\theta^{(1)}\right|_{\tau=t=0}=0$.

The leading order oscillating velocity field, $w$, is given by:

$$
\mathrm{w} \equiv-B \nabla \frac{1}{z} \psi^{(1)}
$$

where $B=\left(-\nabla^{\perp} \theta_{2}, \nabla^{\perp} \theta_{1}\right)=D \theta^{-1}$ and $\theta$ satisfies

$$
\begin{aligned}
& \text { (a) } \bar{\partial}_{t} \theta+\left(\overline{\mathrm{u}} \cdot \nabla_{x}\right) \boldsymbol{\theta}=\mathbf{0}, \\
& \text { (b) }\left.\theta\right|_{t=0}=\mathrm{x} .
\end{aligned}
$$

The pseudo-spectral method is used to solve both the large-scale homogenized equations and the small-scale cell problem. We use the second-order Runge-Kutta method to discretize the equation in time. In our computations, we solve the homogenized equation using a coarse grid and large time step, $\Delta t$. For each coarse grid point within the time interval from $t_{n}$ to $t_{n+1}$ with $t_{n}=n \Delta t$, we solve the cell problem with the periodic boundary condition in the $\mathrm{z}$ variable from $\tau=t_{n} / \epsilon$ to $\tau=t_{n+1} / \epsilon$ using a subgrid $\Delta z$ and a subgrid time step $\Delta \tau$. We then average the cell solution from $\tau=t_{n} / \epsilon$ to $\tau=t_{n+1} / \epsilon$ to evaluate the Reynolds stress term and 
update the mean velocity at $t_{n+1}$. To eliminate the aliasing error in the pseudospectral method, we use a 15th order Fourier smoothing function to damp the high frequency modes.

Below we present numerical results on the decay of 2-D homogeneous turbulence, and compare the homogenization model with well resolved direct numerical simulation (DNS). The DNS uses a $512 \times 512$ fine grid and the same viscosity as the homogenized equation. The simulation starts with random initial condition, where the initial distribution of the stream function in the Fourier space is,

$$
|\hat{\psi}(k)|=\frac{k}{k^{4}+\delta}, \quad k=|\mathbf{k}|,
$$

with random phases. This choice of initial velocity field is similar to the earlier work of Henshaw-Kreiss-Reyna [11]. In the computation, we choose $\delta=10^{-5}$. The initial vorticity distribution is plotted in Figure 1 . At $t=5.0$, coherent vortices

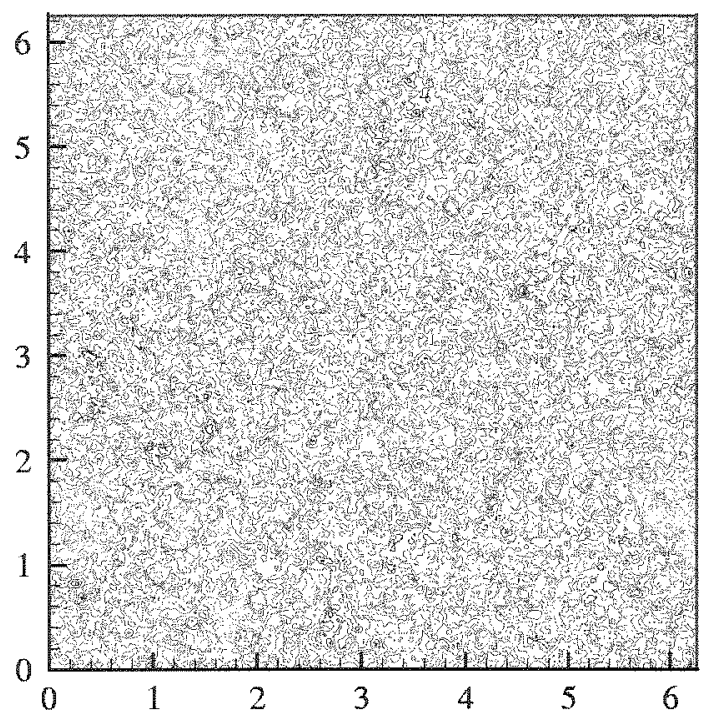

FIGURE 1. Vorticity contour at $t=0$.

emerge from the random initial condition, which is denoted as 'vortex generation period'. At later stages, the flow is dominated by the mutual interactions of coherent vortices. The number of vortices decreases and the averaged vortex radius and circulation increase.

Since our multiscale analysis is developed for well-mixing flows, we start our multiscale computation using the homogenized equations starting at $t=5.0$ when the flow completes the 'vortex generation period'. We use the technique presented in Section 5 to prepare the velocity obtained from the DNS at $t=5.0$ in the form of a two-scale initial condition so that we can apply the homogenized equations (5.1) to solve the multiscale problem. The dimension of the coarse grid (slow variables) 
is $64 \times 64$ and that of small scale (fast variables) is $32 \times 32$. The time step for the homogenized equation is $\Delta t=0.01$ and the subgrid time step for the cell problem is $\Delta \tau=0.01$. Figure 2 compares the vorticity distribution of the homogenization result with DNS result at $t=5.0,10.0$ and 20.0. The plots show the stretching of vortex filament by the mean flow. In addition, the size of the vortices grows due to the merger of vorticity of the same sign, which is one of the mechanisms that contributes to the inverse energy cascade. The vorticity distribution from the homogenization is in excellent agreement with the DNS, suggesting that the multiscale model captures the vortex interactions at both large scales and small scales.

The accuracy of the solution can be examined by studying whether the solution dissipates the correct amount of energy and enstrophy, which are two statistical global quantities of turbulent flows. It is known that the temporal evolution of the mean kinetic energy and the mean enstrophy is governed by the following equations $[12,13]$

$$
\begin{aligned}
& \frac{d}{d t}\left\langle\frac{1}{2}|\mathbf{u}|^{2}\right\rangle=-\nu\left\langle\omega^{2}\right\rangle, \\
& \frac{d}{d t}\left\langle\frac{1}{2} \omega^{2}\right\rangle=-\nu\left\langle\nabla \omega^{2}\right\rangle .
\end{aligned}
$$

From equation (5.8), we can see that the enstrophy is bounded by its initial value. Therefore in the limit of $\nu \rightarrow 0, d\left\langle|\mathbf{u}|^{2}\right\rangle / d t \rightarrow 0$, i.e. the total kinetic energy is conserved. On the other hand, there exists a cascade of enstrophy from large scale to small scale $[\mathbf{1 2}, \mathbf{1 3}]$, which causes the total enstrophy to decay in time. Specifically, vorticity gradients are amplified with the formation of thin filaments. These filaments are stretched until they reach the very small dissipation scales, so that the enstrophy and all positive-order vorticity moments decay. In Figure 3, we show the temporal evolution of the total kinetic energy and the total enstrophy using three different approaches, which are (i) the DNS, (ii) the simulation using the homogenized equations, and (iii) the simulation of the homogenized equations ignoring the Reynolds stress term. With very small viscosity, the energy decay from all 3 simulations is negligible. On the other hand, enstrophy decays continuously, with maximum decay rate during the initial vortex formation period. The decay rate becomes smaller during the vortex merger stage. We can see that the simulation with coarse grid using approach (iii) leads to much slower enstrophy decay because it could not capture the enstrophy cascade from large scales to the dissipation scale. On the other hand, the enstrophy decay rate of the simulation using the homogenized equations is very close to that of the DNS, suggesting that the dissipation mechanism is well resolved within each cell.

We also compare the spectra between the DNS and the simulation using the homogenized equations at $t=20.0$. We reconstruct the fine grid velocity field by

$$
\mathbf{u}^{\epsilon}(t, \mathbf{x}) \approx \mathbf{u}(t, \mathbf{x})+\mathbf{w}\left(t, \boldsymbol{\theta}, \tau, \frac{\boldsymbol{\theta}(\mathbf{t}, \mathbf{x})}{\epsilon}\right)
$$

where the find grid phase function is obtained using the spectral interpolation. The agreement is very good at low wavenumbers. At high wavenumbers, the DNS spectra decay faster than the spectra of the simulation using the homogenized equations. This explains the difference in the enstrophy decay rate between the two simulations. The difference is partly due to neglecting the higher order terms in the homogenized equations and the cell problem. Another more important reason 


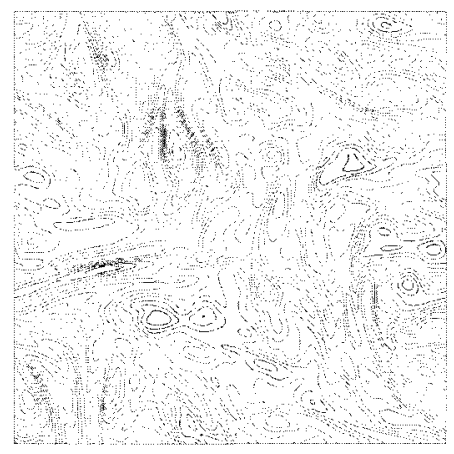

(a) DNS: $t=5.0$

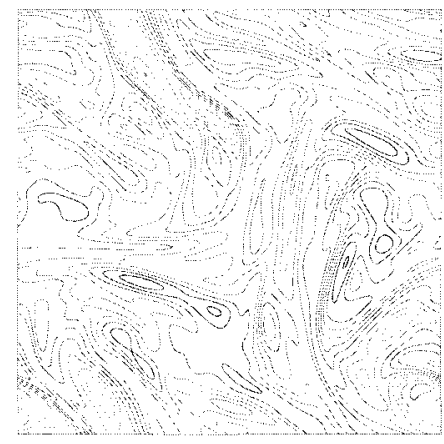

(c) DNS: $\mathbf{t}=10.0$

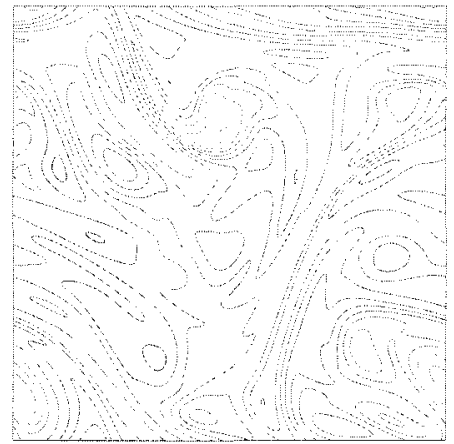

(e) DNS: $\mathrm{t}=20.0$

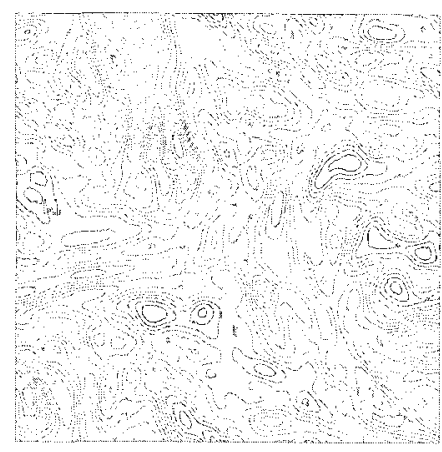

(b) Homogenization: $\mathrm{t}=5.0$

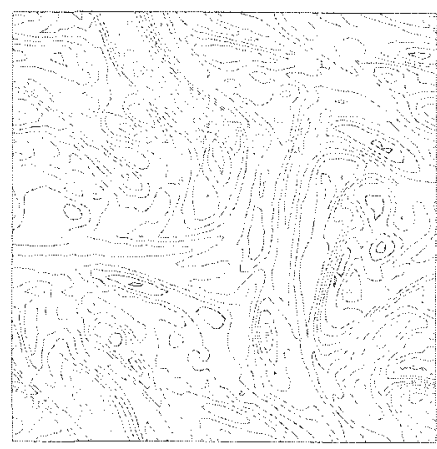

(d) Homogenization: $\mathrm{t}=10.0$

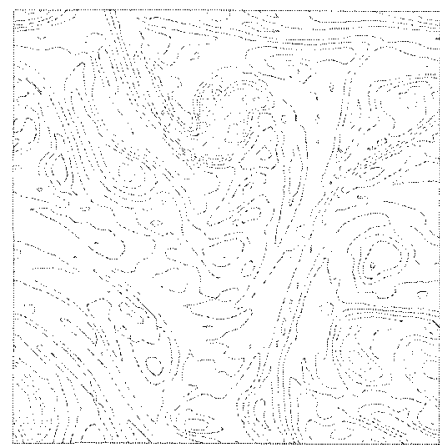

(f) Homogenization: $t=20.0$

Figure 2. Contour plot of vorticity. Contour levels: $\max =1.4$, $\min =-0.9$. 


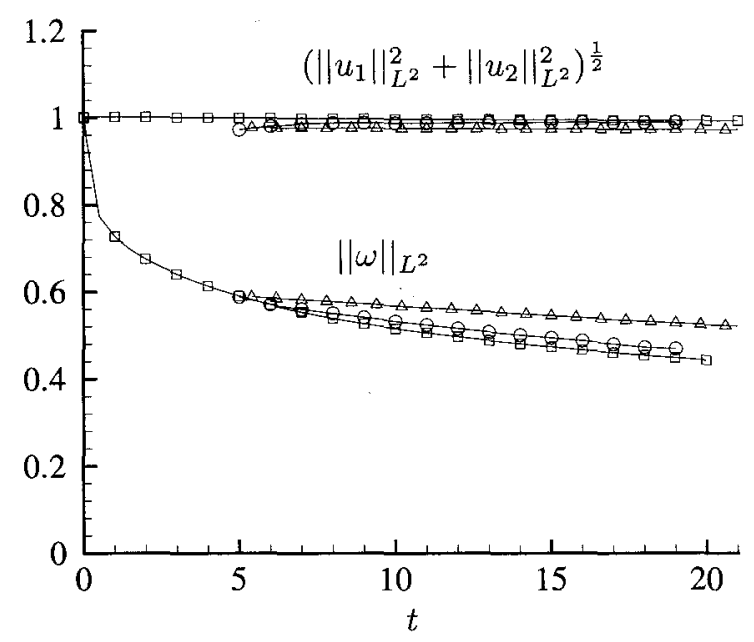

FIgURE 3. Temporal evolution of kinetic energy $\left(\left\|u_{1}\right\|_{L^{2}}^{2}+\right.$ $\left.\left\|u_{2}\right\|_{L^{2}}^{2}\right)^{\frac{1}{2}}$ and enstrophy $\|\omega\|_{L^{2}}(\square)$, DNS(N=512); (०), homogenization; $(\triangle)$, without Reynolds stress terms.

is that there is viscous dissipation in all the scales in the DNS, whereas we neglect viscosity in the cell problem in the homogenized equations. Nonetheless, the model accurately captures the dynamics of the large scale, as well as the averaged effect from the small scales.

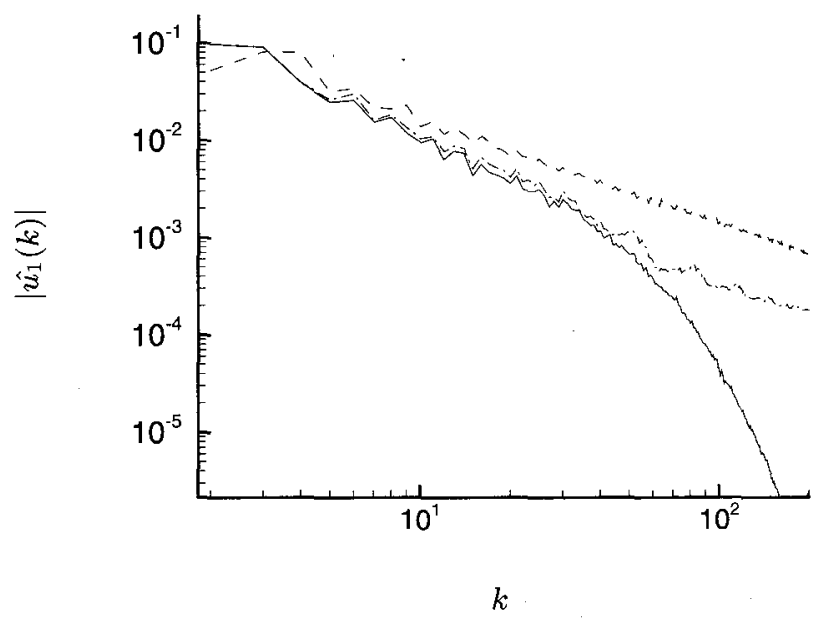

FIGURE 4. Spectrum of velocity component $u_{1} \cdot(----), t=0.0$; $(-)$, DNS, $t=20.0 ;(---)$, homogenization, $t=20.0$. 
5.2. Homogeneous Flows. In this subsection, we discuss how to explore the homogeneity of the flow to reduce the homogenization problem to solving only one cell problem. The MPP model assumes that the perturbations are periodic in the variable $y=\theta / \epsilon$. In [14], Rebollo and Coronil extended the MPP model, and allowed the period cell of the perturbation to be deformed by the meanflow. They derived the $k-\epsilon$ model for locally homogeneous turbulence. In this subsection, we will show how to use a similar technique to solve only one cell problem when the flow is homogeneous.

Note that the original cell problem can be posted as follows

(a) $\partial_{\tau} \widetilde{\mathbf{w}}+\left(\widetilde{\mathbf{w}} \cdot \nabla_{z}\right) \widetilde{\mathbf{w}}+G^{T} G \nabla_{z} q=\mathbf{0}$

(b) $\nabla_{z} \cdot \widetilde{\mathbf{w}}=0$

(c) $\left.\widetilde{\mathbf{w}}\right|_{\tau=t=0}=\mathbf{W}(\mathbf{x}, \mathbf{z})$,

where $\widetilde{\mathbf{w}}=G^{T} \mathbf{w}$ and $G=\nabla_{x} \theta$.

We make a transformation to reduce the above cell problem into a form that can explore the homogeneity of the flow. Specifically, we make the following transformation

$$
\begin{aligned}
& \widetilde{\mathbf{w}}(\mathbf{z}, \tau)=G^{T} \mathbf{w}=G^{T} \mathbf{w}^{*}\left(G^{-T} \mathbf{z}, \tau\right), \\
& q(\mathbf{z}, \tau)=q^{*}\left(G^{-T} \mathbf{z}, \tau\right) .
\end{aligned}
$$

With the above transformation, we reduce the original cell problem (5.10) into the following Euler equations,

(a) $\partial_{\tau} \mathbf{w}^{*}+\left(\mathbf{w}^{*} \cdot \nabla_{z}\right) \mathbf{w}^{*}+\nabla_{z} q^{*}=\mathbf{0}$,

(b) $\nabla_{z} \cdot \mathbf{w}^{*}=0$

(c) $\left.\mathbf{w}^{*}\right|_{\tau=0}=\mathbf{W}(\mathbf{x}, \mathbf{z})$.

Note that unlike the original cell problem (5.10), the transformed cell problem does not depend on the slow variable $\mathbf{x}$ explicitly. The dependence on $\mathbf{x}$ enters only through the initial condition.

The above formulation shows that under the new coordinate system of $\left(\tau, \mathbf{z}^{*}\right)$, with $\mathbf{z}^{*}=G^{-T} \mathbf{z}$, the small cell problem has the same form as the original Euler equations. However, due to the change of coordinates in the fast variable, the periodic structure of the cell problem is now $\mathbf{x}$ dependent. For a matrix $A$, we say a function $f(\mathbf{y})$ to be A-periodic if $f\left(\mathbf{y}^{\prime}\right)$ is periodic where $\mathbf{y}^{\prime}=A \mathbf{y}$. It is clear that, under this transformation, the unit cubic is deformed. A periodic function with respect to $\mathbf{z}$ now becomes a $D \boldsymbol{\theta}^{-1}$-periodic function with respect to $\mathbf{z}^{*}$.

If the initial oscillatory component $\mathbf{w}$ is independent of $\mathbf{x}$, we only need to solve one $\mathbf{w}^{*}\left(\mathbf{z}^{*}, \tau\right)$ using equation (5.12), where $\mathbf{z}^{*}=G^{-T} \mathbf{z}$. The Reynolds stress term appeared in the homogenized equation, $R=\langle\mathbf{w} \otimes \mathbf{w}\rangle$, is given by,

$$
\langle\mathbf{w} \otimes \mathbf{w}\rangle=\left\langle\mathbf{w}^{*}\left(G^{-T} \mathbf{z}, \tau\right) \otimes \mathbf{w}^{*}\left(G^{-T} \mathbf{z}, \tau\right)\right\rangle=\operatorname{det}\left(G^{T}\right)\left\langle\mathbf{w}^{*} \otimes \mathbf{w}^{*}\right\rangle
$$

If $\langle\mathbf{w} \otimes \mathbf{w}\rangle$ is integrated over $[0,1]^{d}$ ( $d$ is the space dimension), $\left\langle\mathbf{w}^{*} \otimes \mathbf{w}^{*}\right\rangle$ should be integrated over the corresponding deformed cell, denoted as $I_{G}$. Therefore, $\langle\mathbf{w} \otimes \mathbf{w}\rangle$ from a different cell corresponds to the average of the same $\mathbf{w}^{*}$ field, but over a different region within the $\mathrm{w}^{*}$ domain. The shape of the region depends on $G^{-T}$. 
If the initial condition is of the form $\left.\mathbf{u}^{\epsilon}\right|_{t=0}=\mathbf{U}(\mathbf{x})+\mathbf{W}\left(\frac{\mathbf{x}}{\epsilon}\right)$, then we need only to solve one cell problem to obtain the Reynolds stress term in the averaged velocity equation

$$
\begin{aligned}
& \text { (a) } \bar{\partial}_{t} \overline{\mathbf{u}}+\left(\overline{\mathbf{u}} \cdot \nabla_{x}\right) \overline{\mathbf{u}}+\nabla_{x} \bar{p}+\nabla_{x} \cdot\langle\mathbf{w} \otimes \mathbf{w}\rangle=\mathbf{0}, \\
& \text { (b) } \nabla_{x} \cdot \overline{\mathbf{u}}=0 \\
& \text { (c) }\left.\overline{\mathbf{u}}\right|_{t=0}=\mathbf{U},
\end{aligned}
$$

where $\mathbf{w}=\mathbf{w}(t, \overline{\boldsymbol{\theta}}, \tau, \mathbf{z}), \mathbf{z}=\overline{\boldsymbol{\theta}} / \epsilon, \tau=t / \epsilon$. For the cell problem, we solve the canonical Euler equation

$$
\begin{aligned}
& \text { (a) } \partial_{\tau} \mathbf{w}^{*}+\left(\mathbf{w}^{*} \cdot \nabla_{y}\right) \mathbf{w}^{*}+\nabla_{y} q^{*}=\mathbf{0}, \\
& \text { (b) } \nabla_{y} \cdot \mathbf{w}^{*}=0 \\
& \text { (c) }\left.\mathbf{w}^{*}\right|_{\tau=0}=\mathbf{W}(\mathbf{y})
\end{aligned}
$$

with periodic boundary condition, where $\mathbf{y}=G^{-T} \mathbf{z}$. At each time step in $t$, the term $\langle\mathbf{w} \otimes \mathbf{w}\rangle$ in equation (5.14) is computed with the solution of $\mathbf{w}^{*}$ at $\tau=t / \epsilon$,

$$
\langle\mathbf{w} \otimes \mathbf{w}\rangle=\int_{[0,1]^{d}} \mathbf{w}^{*}\left(G^{-T} \mathbf{z}, \tau\right) \otimes \mathbf{w}^{*}\left(G^{-T} \mathbf{z}, \tau\right) d \mathbf{z}=\operatorname{det}\left(G^{T}\right) \int_{I_{G}} \mathbf{w}^{*} \otimes \mathbf{w}^{*} d \mathbf{y}
$$

where $I_{G}$ is the deformed cell. Note that this term should be interpolated from $\overline{\boldsymbol{\theta}}$ to $\mathbf{x}$.

For comparison, we also run direct numerical simulations (DNS) in two space dimensions with a resolution of $1024 \times 1024$. The DNS simulations use the same initial velocity field and viscosity. We have to reconstruct the fine grid velocity field from the solution of the homogenized equations by

$$
\mathbf{u}^{\epsilon}(\dot{t}, \mathbf{x}) \approx \mathbf{u}(t, \mathbf{x})+\mathbf{w}\left(t, \boldsymbol{\theta}, \tau, \frac{\boldsymbol{\theta}(\mathbf{t}, \mathbf{x})}{\boldsymbol{\epsilon}}\right),
$$

where $\mathbf{w}\left(t, \boldsymbol{\theta}, \tau, \frac{\boldsymbol{\theta}(\mathbf{t}, \mathbf{x})}{\epsilon}\right)$ is obtained from the homogeneous cell solution $w^{*}\left(y^{*}\right)$ using the mapping function

$$
\mathbf{w}\left(\boldsymbol{\theta}, \frac{\boldsymbol{\theta}(t, \mathbf{x})}{\epsilon}\right)=\mathbf{w}^{*}\left(G^{-T} \frac{\boldsymbol{\theta}(t, \mathbf{x})}{\epsilon}\right)
$$

Next we examine the energy spectra. At small wavenumbers, the initial spectra have slope $O\left(k^{-2}\right)$. The spectra have peaks at mode $n=32$ and its subharmonics, due to the periodic initial oscillation. At later time, the energy spectra will decay to the equilibrium state of $O\left(k^{-3}\right)$ ), and the peaks will be smoothed out due to mixing. Figure 5 compares the energy spectra computed with DNS, the homogenization method using the transformed cell equation. The result shows that at low frequencies, energy spectra from the two methods are almost identical. There is small discrepancy at high wave numbers.

5.3. Numerical Experiments for the 3D Navier-Stokes Equations. In this subsection, we perform careful direct numerical simulations (DNS) of the 3D Navier-Stokes equations to validate the multiscale analysis presented in the previous sections. The DNS is performed using a $256^{3}$ mesh in a periodic $3 \mathrm{D}$ cube of sides $L=2 \pi$. We solve the incompressible Navier-Stokes equation for the velocity field, using a pseudospectral code. Isotropic forcing is added at the large scales ( $|\mathbf{k}|$ between 1 and 2 ). The forcing coefficients were chosen so that the energy 


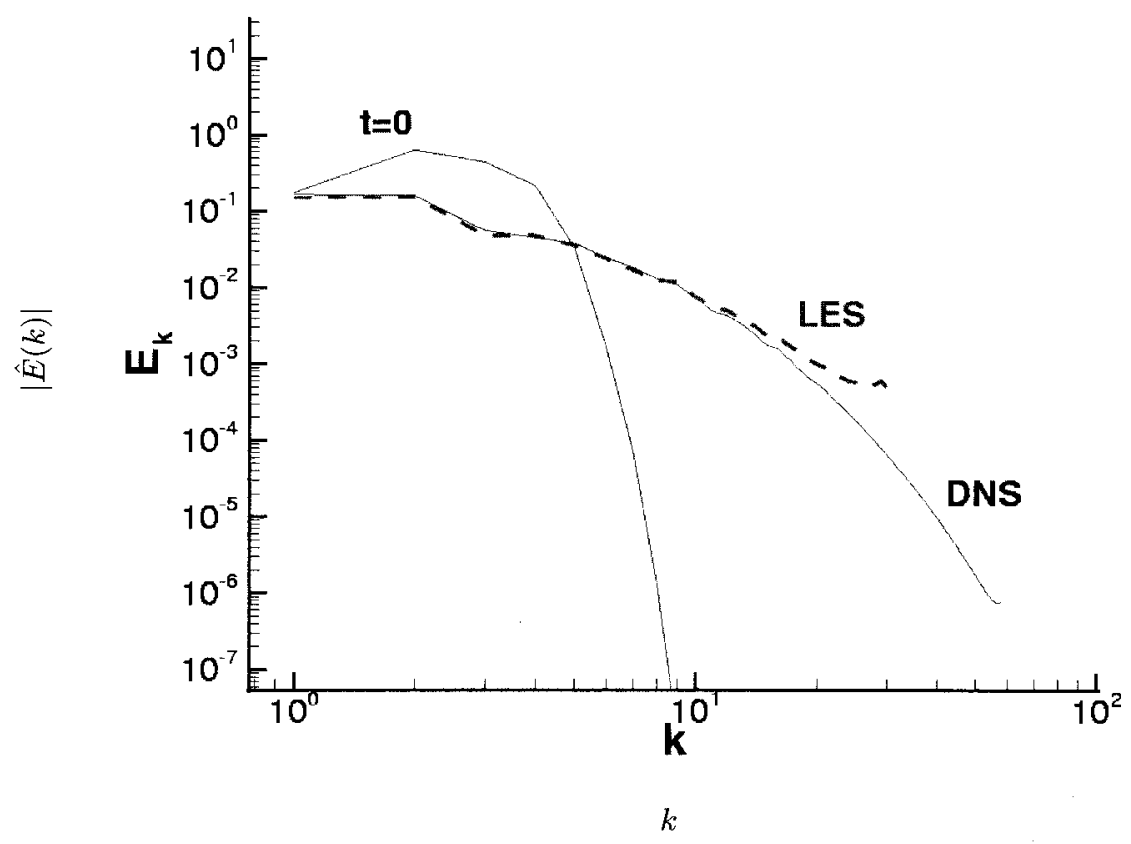

FIgURE 5. Energy spectra.(red), DNS at $t=0.0$; (blue), DNS at $t=10.0 ;$ (black), homogenization with one cell at $t=10.0$.

injection rate $\sum \hat{f}_{i}(\mathbf{k}, \mathbf{t}) \hat{\mathbf{u}}_{\mathbf{i}}(\mathbf{k}, \mathbf{t})$ is constant, where $\hat{f}_{i}(\mathbf{k}, \mathbf{t})$ are the Fourier modes of the forcing function. The injected energy is transported to the small scales due to energy cascade, so the flow becomes statistically stationary in time. A secondorder explicit Runge-Kutta scheme is used for time-marching, and a $2 / 3$ dealiasing method is used for the non-linear terms. The initial condition is a random field with a spectrum peaked around $\left|\mathbf{k}_{\mathbf{p}}\right|=30$.

Figure 6 shows the isosurface of the magnitude of the vorticity at equilibrium $(t=10)$, at a value two standard deviations above the mean value. The intense vorticity is organized into tube-like structures. Figure 7 shows the initial and equilibrium energy spectra. At $t=10.0$, a inertia range is established that has slope $k^{-5 / 3}$. Figure 8 depicts the decay of kinetic energy, due to the forcing and turbulence dissipation. At the beginning, energy dissipation dominates energy injection, and the total kinetic energy decrease. Consequently, dissipation decreases until it reach an equilibrium with the energy injection.

Direct numerical simulation of the homogenized equations for the 3D Euler equations is difficult. It would be too expensive to solve both the averaged equation and the cell problem on every other grid cell. In this paper, we use an alternative approach to examine the accuracy of our multiscale analysis and the Reynolds stress model by utilizing data from the DNS simulation. Specifically, we separate the Navier-Stokes equation into two parts corresponding to two components of the initial condition. The initial condition is separated as the mean part $\mathbf{U}(\mathbf{x})$ (obtained 


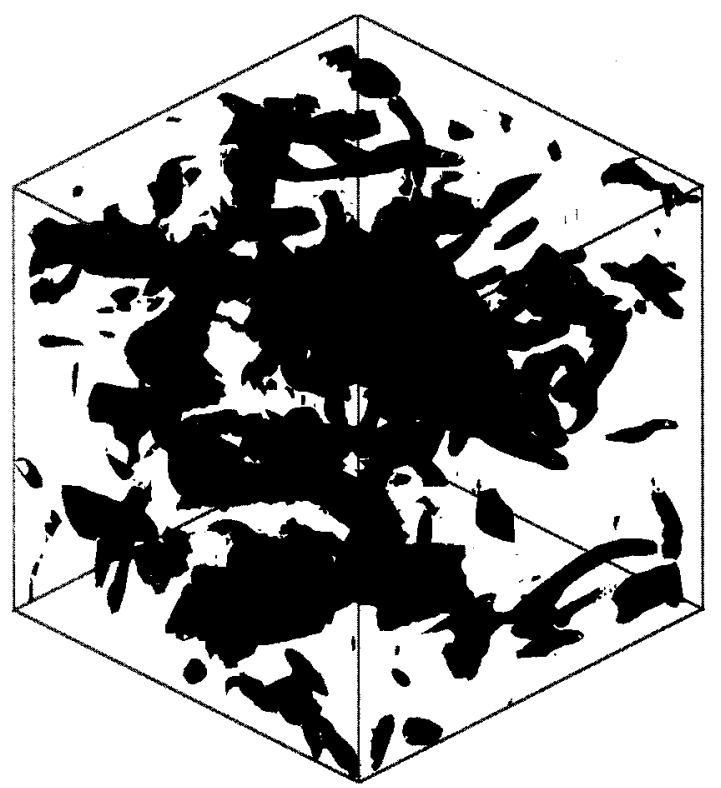

FIGURE 6. Iso-surface plot of vorticity.

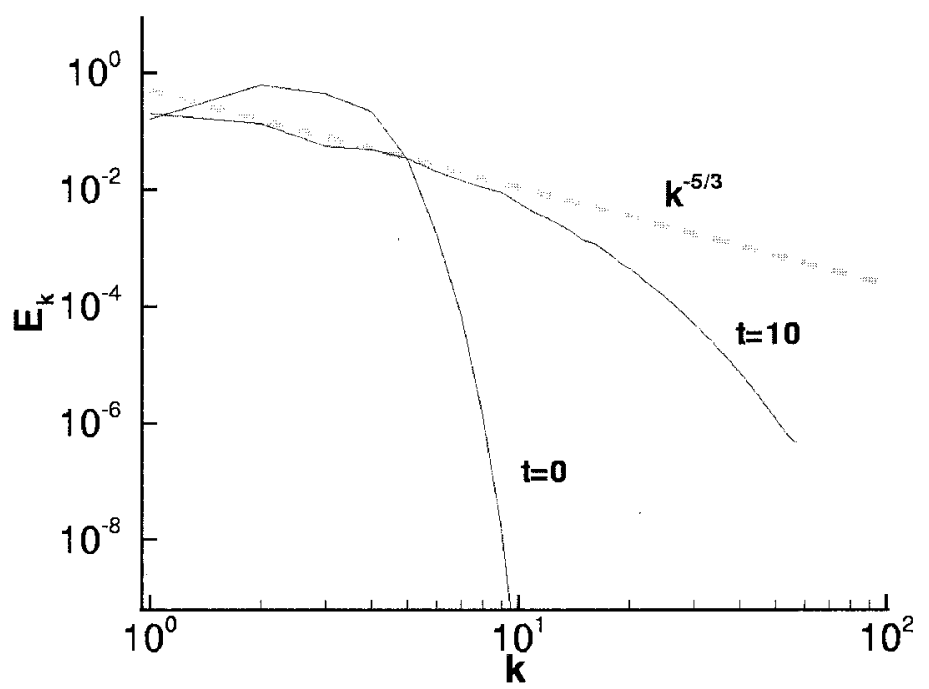

Frgure 7. The initial and equilibrium energy spectra.

by low pass filtering the full velocity field) and oscillatory part $\mathbf{W}(\mathbf{x}, \mathbf{y})$, and then we compute the full velocity field $\mathbf{u}$ and the oscillatory part $\mathbf{w}^{(1)}$ simultaneously. 


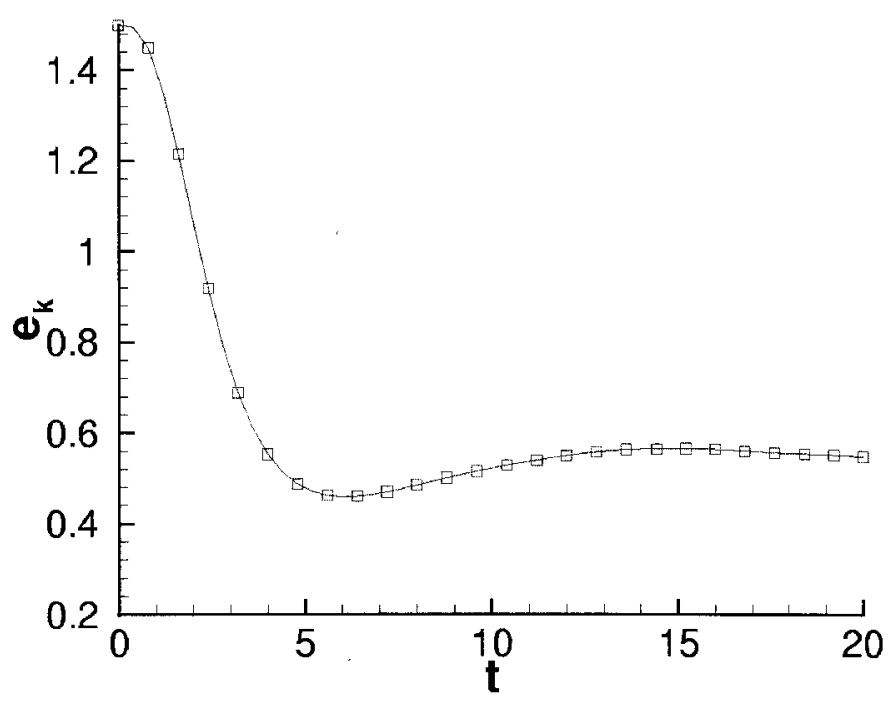

FigurE 8. The decay of kinetic energy.

The governing equation for the full velocity field is given by

$$
\begin{aligned}
& \text { (a) } \partial_{t} \mathbf{u}^{\epsilon}+\left(\mathbf{u}^{\epsilon} \cdot \nabla\right) \mathbf{u}^{\epsilon}=-\nabla p^{\epsilon}+\nu \Delta \mathbf{u}^{\epsilon}, \\
& \text { (b) } \nabla \cdot \mathbf{u}^{\epsilon}=0 \\
& \text { (c) }\left.\mathbf{u}^{\epsilon}\right|_{t=0}=\mathbf{U}+\mathbf{W} .
\end{aligned}
$$

and the governing equation for the oscillatory part corresponding to the initial fluctuation $\mathbf{W}$ is given by

$$
\begin{aligned}
& \text { (a) } \partial_{t} \mathbf{w}^{(1)}+\left(\mathbf{u}^{\epsilon} \cdot \nabla\right) \mathbf{w}^{(1)}=-\nabla q^{(1)}+\nu \Delta \mathbf{w}^{(1)}, \\
& \text { (b) } \nabla \cdot \mathbf{w}^{(1)}=0, \\
& \text { (c) }\left.\mathbf{w}^{(1)}\right|_{t=0}=\mathbf{W} .
\end{aligned}
$$

In the meantime, we compute the Lagrangian map

$$
\begin{aligned}
& \text { (a) } \partial_{t} \boldsymbol{\theta}+\left(\mathbf{u}^{\epsilon} \cdot \nabla\right) \boldsymbol{\theta}=\mathbf{0} \\
& \text { (b) }\left.\boldsymbol{\theta}\right|_{t=0}=\mathbf{x} .
\end{aligned}
$$

The Reynolds stress $\langle\mathbf{w} \otimes \mathbf{w}\rangle]^{*}$ can be constructed from $\mathbf{u}^{\epsilon}, \mathbf{w}^{(1)}$ and $\boldsymbol{\theta}$ using (4.12) and (4.13). On the other hand, the subgrid-stress tensor can be calculated from DNS: $\tau_{i j}=\overline{v_{i} v_{j}}-\bar{v}_{i} \bar{v}_{j}$, where the overline represents the filtering operation. Local accuracy of the Reynolds stress model can be verified by comparison of the Reynolds stress tensor obtained by the two approaches. In addition, the global effect of the Reynolds stress can be tested by plugging the calculated $\langle\mathbf{w} \otimes \mathbf{w}\rangle]^{*}$ into the averaged equation as follows:

$$
\begin{aligned}
& \text { (a) } \partial_{t} \overline{\mathbf{u}}+(\overline{\mathbf{u}} \cdot \nabla) \overline{\mathbf{u}}+\nabla \bar{p}+\nabla_{x} \cdot[\langle\mathbf{w} \otimes \mathbf{w}\rangle]^{*}=\mathbf{0} \\
& \text { (b) } \nabla \cdot \overline{\mathbf{u}}=0 \\
& \text { (c) }\left.\overline{\mathbf{u}}\right|_{t=0}=\mathbf{U}
\end{aligned}
$$


We will then compare the spectra of $\mathbf{u}$ and $\bar{u}$ to check how accurate we can capture the subgrid effect of the small scale solution.

In the present study, we separate the velocity field at equilibrium obtained from DNS into a mean flow $U$ (with $64^{3}$ grid) and an oscillatory part $\mathbb{W}$. At each time step, we solve the averaged Navier-Stokes equations (5.22). The Reynolds stress is calculated using equation (4.12) from the DNS data.

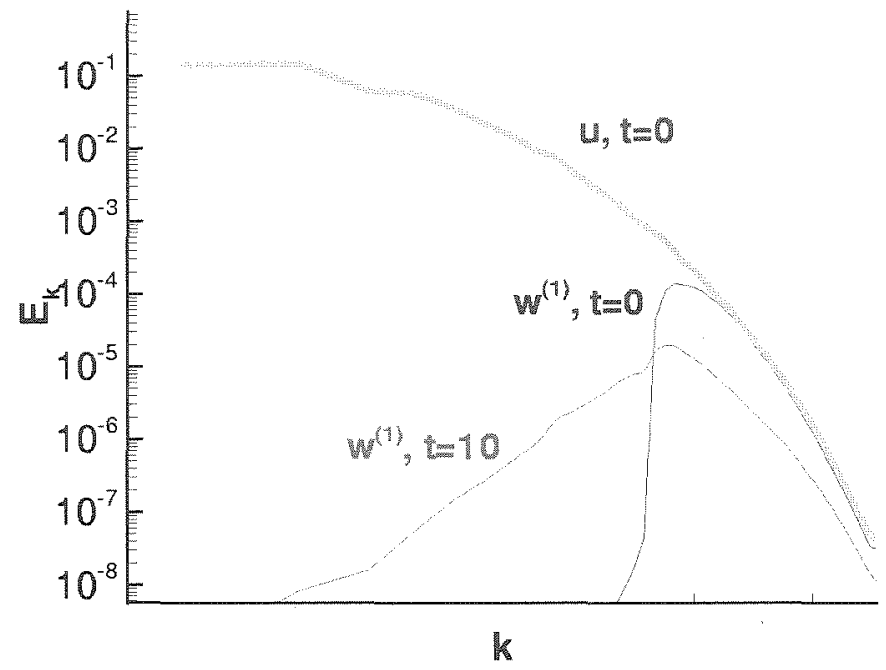

FiguRE 9. The evolution of the spectra of component $\mathbf{w}^{(1)}$.
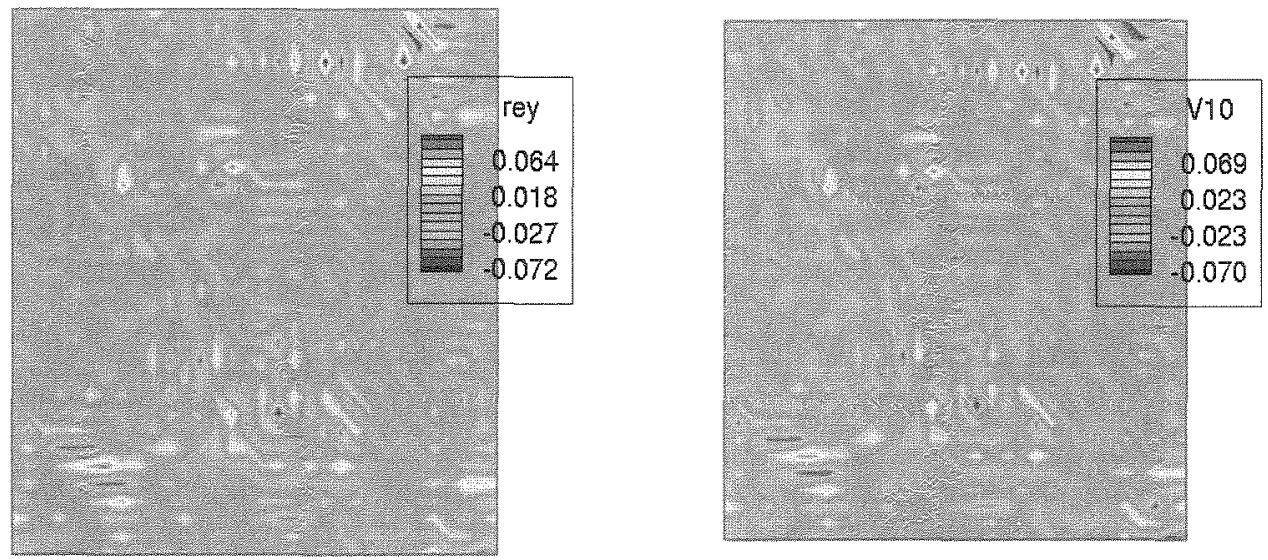

Figure 10. Comparison of Reynolds stress computed with DNS and multiscale model. 


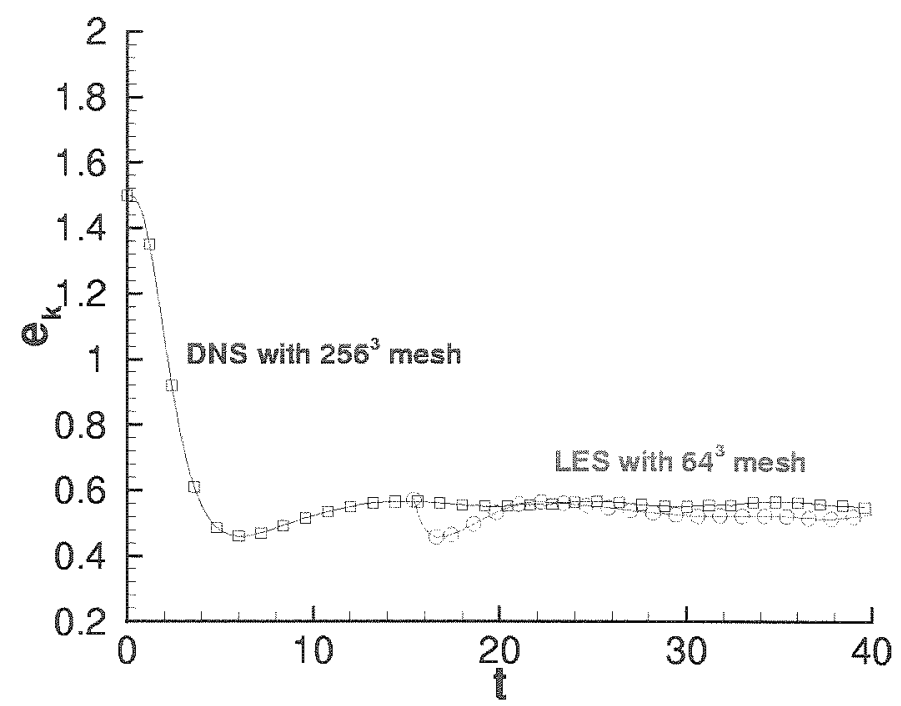

FIGURE 11. The evolution of the total kinetic energy of the DNS and the averaged equations

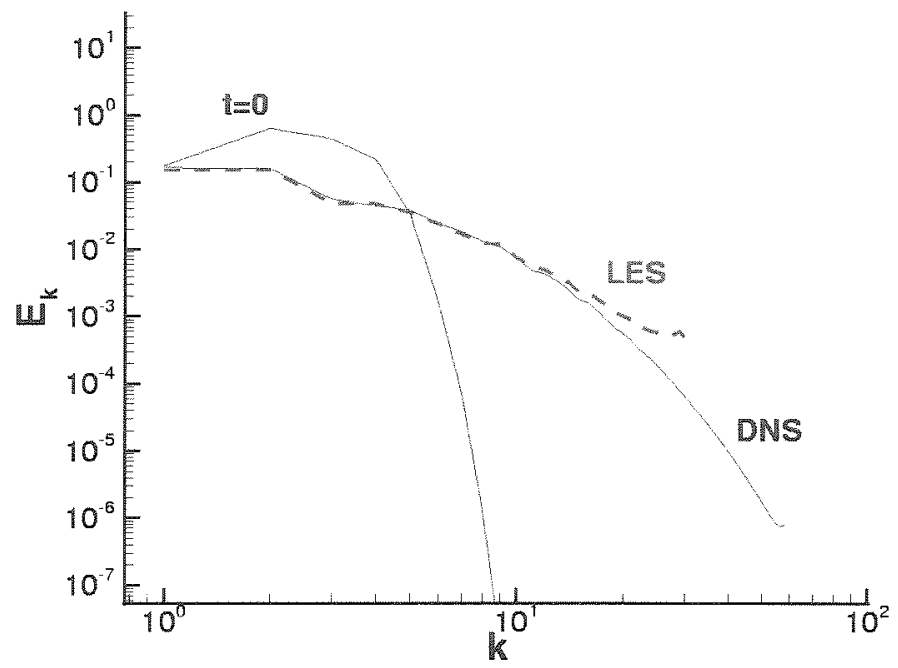

FIGURE 12. the comparison of energy spectra between DNS and the averaged equation at $t=40$.

Figure 9 shows the evolution of the spectra of component $w^{(1)}$, which is driven by the whole velocity field $w^{\epsilon}$. We notice that small scales are generated from the mean flow, and produce the oscillatory component of the sub-grid velocity $\tilde{\mathbf{u}}^{(1)}$. In 
Figure 10, we compare the Reynolds stress computed with DNS and with that computed by the multiscale model. It confirms the accuracy of the multiscale analysis on the Reynolds stress. Figure 11 shows the evolution of the total kinetic energy of the DNS and that from solving the averaged equations (started from $t=15$ ). After an initial transient, the averaged equation establishes the same energy dissipation rate at the cutoff wavenumber, and the flow become statistically stationary. The total kinetic energy is in good agreement with that from the DNS. Next, we compare the energy spectra of the velocity field obtained from DNS and that from the averaged equation at $t=40$. As we can see from Figure 12, the agreement is quite good at low wave numbers. The results show that the Reynolds stress computed from the multiscale model captures the energy cascade from resolved scales to subgrid scales both at local wavenumber and the global spectra.

We are currently investigating how to use the multiscale model presented in this paper to construct an effective coarse grid model. The result will be reported elsewhere in the future.

\section{References}

[1] A. Bensoussan, J. L. Lions, and G. Papanicolaou. Asymptotic Analysis for Periodic Structure. volume 5 of Studies in Mathematics and Its Applications. North-Holland Publ., 1978.

[2] A. Chorin and J. Marsden, A Mathematical Introduction to Fluid Mechanics. Springer, 1983.

[3] U. Frisch, Turbulence: the legacy of A. N. Kolmogorov. Cambridge University Press, New York, 1995.

[4] T. Y. Hou and X. H. Wu, A Multiscale Finite Element Method for Elliptic Problems in Composite Materials and Porous Media, J. Comput. Phys., 134 (1997), 169-189.

[5] A. N. Kolmogorov, Local structure of turbulence in an incompressible fluid at a very high Reynolds number. Dokl. Akad. Nauk SSSR, 30 (1941), 299-302.

[6] D. W. McLaughlin, G. C. Papanicolaou, and O. Pironneau, Convection of Microstructure and Related Problems. SIAM J. Applied Math, 45 (1985), 780-797.

[7] J. Smogorinsky. General circulation experiments with the primitive equations. I. The basic experiment. Monthly Weather Review, 91 (1963), 99-164.

[8] T. Y. Hou, D. P. Yang and K. Wang Homogenization of incompressible Euler equation, J. Comput. Math, 22 (2004), 220-229.

[9] T. Y. Hou, D. P. Yang and H. Ran Multiscale analysis for the $3 D$ incompressible Euler equations, preprint, 2005.

[10] T. Y. Hou, D. P. Yang and H. Ran Multiscale Analysis in Lagrangian Formulation for the 2-D Incompressible Euler Equation, Discrete and Continuous Dynamical Systems, 13, No. 5 , 1153-1186, 2005.

[11] W. D. Henshaw, H. O. Kreiss and L. G. Reyna Smallest Scale Estimates for the Navier-Stokes Equations for Incompressible Fluids, Arch. Ration. Mech. An., 112 (1990), 21-44.

[12] K. G. Batchelor Computation of the Energy Spectrum in Homogeneous Two-Dimensional Turbulence, Phys. Fluids. Suppl. II, 12 (1969), 233-239

[13] R. Kraichnan Inertial Ranges in Two Dimensional Turbulence, Phys. Fluids, 10 (1967), 1417-1423.

[14] T. C. Rebollo and D. F. Coronil Derivation of the $k-\epsilon$ model for locally homogeneous turbulence by homogenization techniques, C.R.Acad. Sci. Paris, Ser. I337 (2003), 431-436. 
Applied and Computational Mathematics, Californta Institute of Technology, PASADENA, CA 91125, USA

E-mail address: hou@acm.caltech.edu

School of Mathematics and System Science, Shandong University, Jinan, 250100, P.R. China.

E-mail address: dpyang@sdu. edu.cn

Applied and Computational Mathematics, California Institute of Technology, PASADENA, CA 91125, USA

E-mail address: ran@acm.caltech.edu 